\title{
ZEROS OF AUTOMORPHIC $L$-FUNCTIONS AND NONCYCLIC BASE CHANGE
}

\author{
JIANYA LIU ${ }^{1}$ and YANGBO YE ${ }^{2}$
}

\begin{abstract}
Let $\pi$ be an automorphic irreducible cuspidal representation of $G L_{m}$ over a Galois (not necessarily cyclic) extension $E$ of $\mathbb{Q}$ of degree $\ell$. Assume that $\pi$ is invariant under the action of the Galois group $\mathrm{Gal}_{E / \mathbb{Q}}$. We computed the $n$-level correlation of normalized nontrivial zeros of $L(s, \pi)$ and proved that it is equal to the $n$-level correlation of normalized nontrivial zeros of a product of $\ell$ distinct $L$-functions $L\left(s, \pi_{1}\right) \cdots L\left(s, \pi_{\ell}\right)$ attached to cuspidal representations $\pi_{1}, \ldots, \pi_{\ell}$ of $G L_{m}$ over $\mathbb{Q}$. This is done unconditionally for $m=1,2$ and for $m=3,4$ with the degree $\ell$ having no prime factor $\leq\left(m^{2}+1\right) / 2$. In other cases, the computation is under a conjecture of bounds toward the Ramanujan conjecture over $E$, and a conjecture on convergence of certain series over prime powers (Hypothesis $\mathrm{H}$ over $E$ and $\mathbb{Q}$ ). The results provide an evidence that $\pi$ should be (noncyclic) base change of $\ell$ distinct cuspidal representations $\pi_{1}, \ldots, \pi_{\ell}$ of $G L_{m}\left(\mathbb{Q}_{\mathbb{A}}\right)$, if it is invariant under the Galois action. A technique used in this article is a version of Selberg orthogonality for automorphic $L$-functions (Lemma 6.2 and Theorem 6.4), which is proved unconditionally, without assuming $\pi$ and $\pi_{1}, \ldots, \pi_{\ell}$ being self-contragredient.
\end{abstract}

1. Introduction. According to Langlands' functoriality conjecture, the $L$-function attached to an automorphic irreducible cuspidal representation $\pi$ of $G L_{m}$ over a number field $E$ should equal a product of $L\left(s, \pi_{j}\right)$ for certain cuspidal representations $\pi_{j}$ of $G L_{m_{j}}$ over $\mathbb{Q}$. Arthur and Clozel [ArtClo] proved that this is indeed the case when $E$ is a cyclic Galois extension of $\mathbb{Q}$ and $\pi$ is stable under $\mathrm{Gal}_{E / \mathbb{Q}}$. In fact in this case, $\pi$ is the base change of exactly $\ell$ nonequivalent cuspidal representations $\pi_{\mathbb{Q}}, \pi_{\mathbb{Q}} \otimes \eta_{E / \mathbb{Q}}, \ldots, \pi_{\mathbb{Q}} \otimes \eta_{E / \mathbb{Q}}^{\ell-1}$ of $G L_{m}\left(\mathbb{Q}_{\mathbb{A}}\right)$, where $\eta_{E / \mathbb{Q}}$ is the nontrivial character of $\mathbb{Q}_{\mathbb{A}}^{\times} / \mathbb{Q}^{\times}$attached to the field extension $E$ according to the class field theory. Consequently (cf. [Bor] and [Lang])

$$
L(s, \pi)=L\left(s, \pi_{\mathbb{Q}}\right) L\left(s, \pi_{\mathbb{Q}} \otimes \eta_{E / \mathbb{Q}}\right) \cdots L\left(s, \pi_{\mathbb{Q}} \otimes \eta_{E / \mathbb{Q}}^{\ell-1}\right)
$$

2000 Mathematics Subject Classification (MSC): 11F70, 11F66, $11 \mathrm{M} 41$.

${ }^{1}$ Partially supported by China NNSF Grant Number 10125101

${ }^{2}$ Project sponsored by the USA NSA under Grant Number MDA904-03-1-0066.

The United States Government is authorized to reproduce and distribute reprints notwithstanding any copyright notation herein. 
where the $L$-functions on the right side are distinct.

When $E$ is noncyclic over $\mathbb{Q}$, factorization of $L(s, \pi)$ into a product of $L$ functions of $G L_{m_{j}}$ over $\mathbb{Q}$ is unknown. Recently, Jianya Liu and Yangbo Ye [LiuYe2] proved that for any $L$-function such a factorization, if exists, must be unique. In particular, we proved that the $L$-function $L\left(s, \pi_{\mathbb{Q}}\right)$, attached to an automorphic irreducible cuspidal representation $\pi_{\mathbb{Q}}$ of $G L_{m}\left(\mathbb{Q}_{\mathbb{A}}\right)$, cannot be factored further as a product of automorphic $L$-functions for $G L_{m_{j}}\left(\mathbb{Q}_{\mathbb{A}}\right)$. In other words, $L\left(s, \pi_{\mathbb{Q}}\right)$ is primitive in the sense of [Sel] (see [Mur1] and [Mur2]).

On the other hand, Rudnick and Sarnak [RudSar] proved that the $n$-level correlation of normalized nontrivial zeros of this $L\left(s, \pi_{\mathbb{Q}}\right)$ follows a GUE model, for a class of test functions whose Fourier transforms have restricted support. Here the zeros are normalized according to their density. For distinct $L$-functions $L\left(s, \pi_{j}\right)$, with $\pi_{j}$ being cuspidal representations of $G L_{m}\left(\mathbb{Q}_{\mathbb{A}}\right), j=1, \ldots, \ell$, Liu and Ye [LiuYe1] proved that the $n$-level correlation of normalized nontrivial zeros of the product $L\left(s, \pi_{1}\right) \cdots L\left(s, \pi_{\ell}\right)$ follows a superposition distribution of individual GUEs from $L\left(s, \pi_{j}\right)$ and products of GUEs of lower ranks, under Selberg's orthogonality conjecture and under a conjecture on the convergence of a sum over prime powers (Hypothesis H: see $\S 2$ ) for $m \geq 5$. Recently, Liu, Wang, and Ye [LiuWangYe] proved this Selberg orthogonality conjecture for automorphic $L$-functions. Therefore, this $n$-level correlation is now known unconditionally for $m \leq 4$ and under the Hypothesis $\mathrm{H}$ for $m \geq 5$. Note that here the test functions also have a restricted support for their Fourier transforms.

In this paper, we will use this GUE correlation to study the factorization of $L(s, \pi)$. We will show that the nontrivial zeros of $L(s, \pi)$ behave in the same way as the nontrivial zeros of $L\left(s, \pi_{1}\right) \cdots L\left(s, \pi_{\ell}\right)$.

TheOrem 1.1. Let $E$ be a Galois extension of $\mathbb{Q}$ of degree $\ell$, and $\pi$ an automorphic irreducible cuspidal representation of $G L_{m}\left(E_{\mathbb{A}}\right)$ with unitary central character. Assume (i) Hypothesis $H$ over $E$ and over $\mathbb{Q}$ when $m \geq 5$ and (ii) Conjecture 2.1 when $m \geq 3$. Suppose that $\pi \cong \pi^{\sigma}$ for all $\sigma \in G a l_{E / \mathbb{Q}}$. Then the $n$-level correlation of normalized nontrivial zeros of $L(s, \pi)$ is equal to the n-level correlation of normalized nontrivial zeros of a product of $\ell$ distinct automorphic $L$-functions attached to cuspidal representations of $G L_{m}\left(\mathbb{Q}_{\mathbb{A}}\right)$.

Note that Theorem 1.1 is an unconditional result for $m=1$ and 2. For $m=3$ and 4 , it is also unconditional when $(30, \ell)=1$ and $(210, \ell)=1$, respectively. See $\S 2$ for details.

Theorem 1.1 provides an evidence that $L(s, \pi)$ should factor into a product of $L$-functions $\ell$ distinct automorphic $L$-functions attached to cuspidal representations of $G L_{m}\left(\mathbb{Q}_{\mathbb{A}}\right)$, and suggests that the base change and factorization of $L$-functions such as in (1.1) should hold for noncyclic extension fields as well.

We remark that our results contain much less information than what was achieved in [ArtClo], as we cannot see individual representations $\pi_{\mathbb{Q}}, \ldots, \pi_{\mathbb{Q}} \otimes$ 
$\eta_{E / \mathbb{Q}}^{\ell-1}$ as in (1.1). Since we are seeking less information, we can determine through zero distributions the base change structure of $\pi$ beyond the scope of [ArtClo].

The reason behind this is indeed the universality of the $n$-level correlation of zeros discussed in [RudSar]: The $n$-level correlation of normalized nontrivial zeros of $L\left(s, \pi_{\mathbb{Q}}\right)$ is independent of $\pi_{\mathbb{Q}}$, as long as it is a cuspidal representation of $G L_{m}\left(\mathbb{Q}_{\mathbb{A}}\right)$.

Our computation of $n$-level correlation will be carried our for test functions $f$ whose Fourier transforms $\Phi(\xi)$ as in (2.8) below have restricted support $\sum_{1 \leq j \leq n}\left|\xi_{j}\right|<2 / m$. This restriction of support is good enough for our purpose. We will not assume conjectures that the $n$-level GUE correlation holds for test functions with arbitrary support of their Fourier transforms.

One of the main techniques used in the computation is a version of Selberg orthogonality [Sel] for automorphic $L$-functions. First we proved a Merten's theorem for Rankin-Selberg $L$-functions $L\left(s, \pi \times \tilde{\pi}^{\prime}\right)$ (Lemma 6.2 and Theorem 6.4). The proof is unconditional, and $\pi$ and $\pi^{\prime}$ are cuspidal representations of $G L_{m}\left(E_{\mathbb{A}}\right)$ not necessarily self-contragredient. Next we use Hypothesis $\mathrm{H}$ to control sums over prime powers and use Conjecture 2.1 to control sums over primes which do not split completely in $E$. The resulting orthogonality (Lemmas 7.1 and 7.3) is then for sums over completely split primes. By an argument in [Rog], information on $\pi \cong \pi^{\sigma}$ or not can be characterized by equivalence relationships among local representations $\pi_{v}$ at places $v$ lying above completely split primes. This information will therefore be described by orthogonality, and eventually by the $n$-level correlation of normalized nontrivial zeros.

2. Notation and assumption. Let $E$ be a Galois number field of degree $\ell$ over $\mathbb{Q}$. Let $E_{\mathbb{A}}=\prod_{v}^{\prime} E_{v}$ be its adele ring, where $v$ goes over all places of $E$, and $\Pi^{\prime}$ denotes a restricted product. For any prime $p$, we have $E \otimes \mathbb{Q} \mathbb{Q}_{p}=\bigoplus_{v \mid p} E_{v}$, where $v$ with $v \mid p$ are places of $E$ lying above $p$. Since $E$ is Galois over $\mathbb{Q}$, all $E_{v}$ with $v \mid p$ are isomorphic. Denote by $\ell_{p}$ the degree, by $e_{p}=\operatorname{ord}_{v_{i}}(p)$ the order of ramification, and by $f_{p}$ the modular degree of $E_{v}$ over $\mathbb{Q}$ for $v \mid p$. Then $\ell_{p}=e_{p} f_{p}$ and $q_{v}=p^{f_{p}}$ is the module of $E_{v}$. On the other hand, $E \otimes_{\mathbb{Q}} \mathbb{R}$ is either $=\bigoplus_{v \mid \infty} \mathbb{R}$, or $=\bigoplus_{v \mid \infty} \mathbb{C}$.

Let $\pi$ be an automorphic irreducible cuspidal representation of $G L_{m}\left(E_{\mathbb{A}}\right)$ with unitary central character. The (finite part) $L$-function attached to $\pi$ is given by the Euler product $L(s, \pi)=\prod_{v<\infty} L\left(s, \pi_{v}\right)$ for $\operatorname{Re} s>1$. The definition of local factor $L\left(s, \pi_{v}\right)$ is given by

$$
L\left(s, \pi_{v}\right)=\prod_{1 \leq j \leq m}\left(1-\alpha_{\pi}(j, v) q_{v}^{-s}\right)^{-1}
$$

where $\alpha_{\pi}(j, v), 1 \leq j \leq m$, are complex numbers given by the Langlands correspondence. For any prime $p$, denote by $L_{p}(s, \pi)=\prod_{v \mid p} L\left(s, \pi_{v}\right)$ the product of 
local factors above $p$. Then

$$
L_{p}(s, \pi)=\prod_{v \mid p} \prod_{1 \leq j \leq m}\left(1-\alpha_{\pi}(j, v) p^{-f_{p} s}\right)^{-1} .
$$

Similarly, the product of Archimedean local factors is given by $L_{\infty}(s, \pi)=$ $\prod_{v \mid \infty} L\left(s, \pi_{v}\right)$, where

$$
L\left(s, \pi_{v}\right)=\prod_{1 \leq j \leq m} \Gamma_{\mathbb{R}}\left(s+\mu_{\pi}(j, v)\right)
$$

if $v$ is real, and

$$
L\left(s, \pi_{v}\right)=\prod_{1 \leq j \leq m} \Gamma_{\mathbb{C}}\left(s+\mu_{\pi}(j, v)\right)
$$

if $v$ is complex. Here $\mu_{\pi}(j, v)$ are again complex numbers given by the Langlands correspondence, $\Gamma_{\mathbb{R}}(s)=\pi^{-s / 2} \Gamma(s / 2)$, and $\Gamma_{\mathbb{C}}(s)=(2 \pi)^{-s} \Gamma(s)$.

We will need a bound for $\alpha_{\pi}(j, v)$ :

$$
\left|\alpha_{\pi}(j, v)\right| \leq p^{f_{p}\left(1 / 2-1 /\left(\ell m^{2}+1\right)\right)}
$$

This bound holds for any $\pi_{v}$, either ramified or unramified. It was first observed by Serre [Ser] and appeared in published form in [LuoRudSar]. A complete proof is given in [RudSar] for the case of $E=\mathbb{Q}$, using an argument of Landau [Land].

When $\pi_{v}$ is unramified, the generalized Ramanujan conjecture claims that

$$
\left|\alpha_{\pi}(j, v)\right|=1
$$

The best known bounds toward this Ramanujan conjecture over an arbitrary number field are

$$
\left|\alpha_{\pi}(j, v)\right| \leq q_{v}^{1 / 9}=p^{f_{p} / 9}
$$

for $m=2([$ KimShah $])$, and

$$
\left|\alpha_{\pi}(j, v)\right| \leq q_{v}^{1 / 2-1 /\left(m^{2}+1\right)}=p^{f_{p}\left(1 / 2-1 /\left(m^{2}+1\right)\right)}
$$

for general $m$ ([LuoRudSar]), where $v \mid p$.

We will not assume the generalized Ramanujan conjecture, but assume a bound $\theta_{p}$ toward it for any $p$ which is unramified and does not split completely in $E$. 
Conjecture 2.1. For any $p$ which is unramified and does not split completely in $E$, we have for any $v \mid p$ that

$$
\left|\alpha_{\pi}(j, v)\right| \leq q_{v}^{\theta_{p}}=p^{f_{p} \theta_{p}}
$$

where $\theta_{p}=1 / 2-1 /\left(2 f_{p}\right)-\varepsilon$ for a small $\varepsilon>0$.

We remark that $e_{p}=1$ and hence $f_{p}=\ell_{p}$ when $p$ is unramified. Since $p$ does not split completely in $E$, we know that $f_{p} \geq 2$. Consequently Conjecture 2.1 is known for $m=2$, according to (2.3). Conjecture 2.1 is trivial for $m=1$. Recall that $f_{p} \mid \ell$. Thus conjecture 2.1 is known when all prime factors of $\ell$ are $>\left(m^{2}+1\right) / 2$. For $m=3$ this means that any $p \mid \ell$ is $\geq 7$, while for $m=4$, Conjecture 2.1 is true when any $p \mid \ell$ is $\geq 11$.

We also need the Hypothesis $\mathrm{H}$ ([RudSar]) generalized to $E$.

Hypothesis H. Let $\pi$ be an automorphic irreducible cuspidal representation of $G L_{m}\left(E_{\mathbb{A}}\right)$ with unitary central character. Then for any fixed $k \geq 2$

$$
\sum_{p} \frac{\log ^{2} p}{p^{k f_{p}}} \sum_{v \mid p}\left|\sum_{1 \leq j \leq m} \alpha_{\pi}^{k}(j, v)\right|^{2}<\infty .
$$

We note that Hypothesis $\mathrm{H}$ is an easy consequence of the generalized Ramanujan conjecture (2.2). Since there are only finitely many $p$ which are not unramified in $E$, the sum in (2.5) may be taken over all unramified $p$. As we have assumed Conjecture 2.1, we know that

$$
\begin{aligned}
& \sum_{\substack{p \text { unramified, } \\
\text { not split completely }}} \frac{\log ^{2} p}{p^{k f_{p}}} \sum_{v \mid p}\left|\sum_{1 \leq j \leq m} \alpha_{\pi}^{k}(j, v)\right|^{2} \\
\ll & \sum_{\substack{p \text { unramified, } \\
\text { not split completely }}} \frac{\log ^{2} p}{p^{k f_{p}}} p^{2 k f_{p} \theta_{p}}<\infty .
\end{aligned}
$$

Consequently under Conjecture 2.1, Hypothesis $\mathrm{H}$ claims that for any fixed $k \geq 2$

$$
\sum_{p \text { splits completely }} \frac{\log ^{2} p}{p^{k}} \sum_{v \mid p}\left|\sum_{1 \leq j \leq m} \alpha_{\pi}^{k}(j, v)\right|^{2}<\infty .
$$

As in the case of $E=\mathbb{Q}$, Hypothesis $\mathrm{H}$ is trivial for $m=1$. For $m=2$, it can be proved using the bound in (2.3). In fact, (2.3) implies that $\left|\alpha_{\pi}^{k}\left(j, v_{i}\right)\right| \leq p^{k f_{p} / 9}$ and hence

$$
\sum_{p} \frac{\log ^{2} p}{p^{k f_{p}}} \sum_{v \mid p}\left|\sum_{1 \leq j \leq m} \alpha_{\pi}^{k}(j, v)\right|^{2} \ll \sum_{p} \frac{\log ^{2} p}{p^{7 k / 9}}<\infty
$$

when $k \geq 2$. In Appendix, we will prove Hypothesis $\mathrm{H}$ for $m=3$. For $m=$ 4, Hypothesis $\mathrm{H}$ is a consequence of [Kim], Proposition 6.2, as pointed out by [KimSar]. 
Let $g_{j}$ be a compactly supported smooth function on $\mathbb{R}$. Then its Fourier transform

$$
h_{j}(r)=\int_{\mathbb{R}} g_{j}(u) e^{i r u} d u
$$

is entire and rapidly decreasing on $\mathbb{R}$. We denote $\mathbf{h}=\left(h_{1}, \cdots, h_{n}\right)$ and define

$$
\kappa(\mathbf{h})=\int_{\mathbb{R}} h_{1}(r) \cdots h_{n}(r) d r .
$$

Given $\Phi \in C_{c}^{1}\left(\mathbb{R}^{n}\right)$ we define

$$
f(x)=\int_{\mathbb{R}^{n}} \Phi(\xi) \delta\left(\xi_{1}+\cdots+\xi_{n}\right) e(-x \cdot \xi) d \xi,
$$

where $x=\left(x_{1}, \ldots, x_{n}\right), \xi=\left(\xi_{1}, \ldots, \xi_{n}\right), x \cdot \xi=x_{1} \xi_{1}+\cdots+x_{n} \xi_{n}, \delta(t)$ is the Dirac mass at zero, and $e(t)=e^{2 \pi i t}$.

The $n$-level correlation of normalized nontrivial zeros of the $L$-function $L(s, \pi)$ is given by

$$
\sum_{\substack{i_{1}, \ldots, i_{n} \\ \text { distinct }}} h_{1}\left(\frac{\gamma_{i_{1}}}{T}\right) \cdots h_{n}\left(\frac{\gamma_{i_{n}}}{T}\right) f\left(\frac{\gamma_{i_{1}} m \log T}{2 \pi}, \cdots, \frac{\gamma_{i_{n}} m \log T}{2 \pi}\right),
$$

where the sum is taken over distinct indices $i_{1}, \ldots, i_{n}$, of nontrivial zeros $\rho_{i_{\nu}}=$ $(1 / 2)+i \gamma_{i_{\nu}}, \nu=1, \ldots, n$, of $L(s, \pi)$. Without assuming the Riemann Hypothesis, $\gamma_{i_{j}}$ are complex numbers. Here the factor $m \log T /(2 \pi)$ provides the normalization for zeros $\rho_{i_{\nu}}$. In the following sections, we will first compute the same sum as in (2.9) but taken over all indices of nontrivial zeros. We will denote this latter sum by

$$
\sum_{\gamma_{1}, \cdots, \gamma_{n}} h_{1}\left(\frac{\gamma_{1}}{T}\right) \cdots h_{n}\left(\frac{\gamma_{n}}{T}\right) f\left(\frac{\gamma_{1} m \log T}{2 \pi}, \cdots, \frac{\gamma_{n} m \log T}{2 \pi}\right) .
$$

There is an argument in [RudSar] and [LiuYe1] which we will use to deduce (2.9) from (2.10).

\section{The main theorems.}

TheOrem 3.1. Let $E$ be a Galois extension of $\mathbb{Q}$ of degree $\ell$, and $\pi$ an automorphic irreducible cuspidal representation of $G L_{m}\left(E_{\mathbb{A}}\right)$ with unitary central character. Let $a, 1 \leq a \leq \ell$, be the number of elements $\sigma \in G a l_{E / \mathbb{Q}}$ with $\pi \cong \pi^{\sigma}$. 
Assume $m \leq 4$ or Hypothesis $H$ over $E$ for $m \geq 5$. Also assume Conjecture 2.1 when $m \geq 3$ and there is $p \mid \ell$ such that $p \leq\left(m^{2}+1\right) / 2$. Then

$$
\begin{aligned}
& \sum_{\gamma_{1}, \cdots, \gamma_{n}} h_{1}\left(\frac{\gamma_{1}}{T}\right) \cdots h_{n}\left(\frac{\gamma_{n}}{T}\right) f\left(\frac{\gamma_{1} m \log T}{2 \pi}, \ldots, \frac{\gamma_{n} m \log T}{2 \pi}\right) \\
= & \frac{\kappa(\mathbf{h})}{2 \pi} m T(\log T) \ell^{n} \Phi(0, \cdots, 0) \\
& +\frac{\kappa(\mathbf{h})}{2 \pi} m T(\log T) \sum_{1 \leq r \leq n / 2} \frac{n ! \ell^{n-2 r} a^{r}}{2^{r} r !(n-2 r) !} \int_{\mathbb{R}^{r}}\left|v_{1}\right| \cdots\left|v_{r}\right| \\
& \times \Phi\left(v_{1}, \ldots, v_{r},-v_{1}, \ldots,-v_{r}, 0, \ldots, 0\right) d v_{1} \cdots d v_{r}+O(T),
\end{aligned}
$$

where the sum on the left side is taken over all indices of zeros $\rho_{j}=1 / 2+$ $i \gamma_{j}, j=1, \ldots, n$, of $L(s, \pi)$. Here $g_{j} \in C_{c}^{\infty}(\mathbb{R}), \Phi \in C_{c}^{1}\left(\mathbb{R}^{n}\right)$ is supported in $\sum_{1 \leq j \leq n}\left|\xi_{j}\right|<2 / m$, while $h_{j}, \kappa(\mathbf{h})$, and $f$ are given in (2.6) through (2.8), respectively.

When $a=\ell$, i.e., when $\pi$ is invariant under the action of $\mathrm{Gal}_{E / \mathbb{Q}}$, we can apply the results of an argument of combinatorial sieving in [LiuYe1], §9, directly to (3.1). A set partition $\underline{H}$ of $\underline{N}=(1, \cdots, n)$ is a decomposition of $\underline{N}$ into disjoint subsets $\underline{H}=\left[H_{1}, \cdots, H_{\nu}\right]$, where $\nu=\nu(\underline{H})$ is the number of subsets in $\underline{H}$. For a given set partition $\underline{H}$, define

$$
W \frac{H}{n}\left(x_{1}, \ldots, x_{n}\right)=\prod_{1 \leq k \leq \nu(\underline{H})} \operatorname{det}\left(K\left(x_{i}-x_{j}\right)\right)_{i, j \in H_{k}}
$$

where $K(x)=(\sin \pi x) /(\pi x)$ if $x \neq 0$, and $K(x)=1$ if $x=0$.

COROLlARY 3.2. With notation and assumption as in Theorem 3.1, we assume that $\pi$ is invariant under the action of $G_{E l} / \mathbb{Q}$. Then the $n$-level correlation of normalized nontrivial zeros of $L(s, \pi)$ is given by

$$
\begin{aligned}
& \sum_{\substack{i_{1}, \ldots, i_{n} \\
\text { distinct }}} h_{1}\left(\frac{\gamma_{i_{1}}}{T}\right) \cdots h_{n}\left(\frac{\gamma_{i_{n}}}{T}\right) f\left(\frac{\gamma_{i_{1}} m \log T}{2 \pi}, \ldots, \frac{\gamma_{i_{n}} m \log T}{2 \pi}\right) \\
= & \frac{\kappa(\mathbf{h})}{2 \pi} m T(\log T) \sum_{\substack{\left.\frac{H}{H}\right) \\
\nu \ell}}\left(\begin{array}{c}
\ell \\
\nu(\underline{H})
\end{array}\right) \\
& \times \int_{\mathbb{R}^{n}} f(x) W^{\underline{H}}(x) \delta\left(\frac{x_{1}+\cdots+x_{n}}{n}\right) d x_{1} \cdots d x_{n}+O(T),
\end{aligned}
$$

where the sum on the left side is taken over distinct indices of zeros $\rho_{i_{j}}=1 / 2+$ $i \gamma_{i_{j}}, j=1, \ldots, n$, of $L(s, \pi)$.

The following theorem and its corollary were proved in [LiuYe1] and, for the removal of the assumption on Selberg's orthogonality conjecture, in [LiuWangYe]. 
THEOREM 3.3. Let $\pi_{1}, \ldots, \pi_{\ell}$ be automorphic irreducible cuspidal representations of $G L_{m}\left(\mathbb{Q}_{\mathbb{A}}\right)$ with unitary central character, such that $\pi_{i} \neq \pi_{j}$ for any $i \neq j$. Assume $m \leq 4$ or Hypothesis $H$ over $\mathbb{Q}$ for $m \geq 5$. Then

$$
\begin{aligned}
& \sum_{\gamma_{1}, \ldots, \gamma_{n}} h_{1}\left(\frac{\gamma_{1}}{T}\right) \cdots h_{n}\left(\frac{\gamma_{n}}{T}\right) f\left(\frac{\gamma_{1} m \log T}{2 \pi}, \ldots, \frac{\gamma_{n} m \log T}{2 \pi}\right) \\
= & \frac{\kappa(\mathbf{h})}{2 \pi} m T(\log T) \ell^{n} \Phi(0, \ldots, 0) \\
& +\frac{\kappa(\mathbf{h})}{2 \pi} m T(\log T) \sum_{1 \leq r \leq n / 2} \frac{n ! \ell^{n-r}}{2^{r} r !(n-2 r) !} \int_{\mathbb{R}^{r}}\left|v_{1}\right| \cdots\left|v_{r}\right| \\
& \times \Phi\left(v_{1}, \ldots, v_{r},-v_{1}, \ldots,-v_{r}, 0, \ldots, 0\right) d v_{1} \cdots d v_{r}+O(T),
\end{aligned}
$$

where the sum on the left side is taken over all indices of zeros $\rho_{j}=1 / 2+i \gamma_{j}$, $j=1, \ldots, n$, of $L\left(s, \pi_{1}\right) \cdots L\left(s, \pi_{\ell}\right)$. Here $g_{j} \in C_{c}^{\infty}(\mathbb{R}), \Phi \in C_{c}^{1}\left(\mathbb{R}^{n}\right)$ is supported in $\sum_{1 \leq j \leq n}\left|\xi_{j}\right|<2 / m$, while $h_{j}, \kappa(\mathbf{h})$, and $f$ are given in (2.6) through (2.8), respectively.

Corollary 3.4. Assume $m \leq 4$ or Hypothesis $H$ over $\mathbb{Q}$ for $m \geq 5$. With the same notion as in Theorem 3.3, the n-level correlation of normalized nontrivial zeros of $L\left(s, \pi_{1}\right) \cdots L\left(s, \pi_{\ell}\right)$ is given by

$$
\begin{aligned}
& \sum_{\substack{i_{1}, \ldots, i_{n} \\
\text { distinct }}} h_{1}\left(\frac{\gamma_{i_{1}}}{T}\right) \cdots h_{n}\left(\frac{\gamma_{i_{n}}}{T}\right) f\left(\frac{\gamma_{i_{1}} m \log T}{2 \pi}, \ldots, \frac{\gamma_{i_{n}} m \log T}{2 \pi}\right) \\
= & \frac{\kappa(\mathbf{h})}{2 \pi} m T(\log T) \sum_{\substack{\underline{H} \\
\nu(\underline{H}) \leq \ell}}\left(\begin{array}{c}
\ell \\
\nu(\underline{H})
\end{array}\right) \\
& \times \int_{\mathbb{R}^{n}} f(x) W \frac{H}{n}(x) \delta\left(\frac{x_{1}+\cdots+x_{n}}{n}\right) d x_{1} \cdots d x_{n}+O(T),
\end{aligned}
$$

where the sum on the left side is taken over distinct indices of zeros $\rho_{i_{j}}=1 / 2+$ $i \gamma_{i_{j}}, j=1, \ldots, n$, of $L\left(s, \pi_{1}\right) \cdots L\left(s, \pi_{\ell}\right)$.

Comparing the right side of (3.2) and (3.4), we conclude that the $n$-level correlation of normalized nontrivial zeros of $L(s, \pi)$ equals the $n$-level correlation of normalized nontrivial zeros of a product of $\ell$ distinct $L$-functions attached to cuspidal representations $\pi_{1}, \ldots, \pi_{\ell}$ of $G L_{m}$ over $\mathbb{Q}$, when $\pi$ is invariant under the Galois action. This is the proof of Theorem 1.1. In the following sections we will prove Theorem 3.1.

4. $L$-functions. Again let $E$ be a Galois number field of degree $\ell$ over $\mathbb{Q}$. Denote $\Phi(s, \pi)=L_{\infty}(s, \pi) L(s, \pi)$, for Re $s>1$. Then by a classical result of 
[GodJac], $\Phi(s, \pi)$ extends to an entire function with the exception of $\zeta(s)$, which has a simple pole at $s=1$. $\Phi(s, \pi)$ also has a functional equation

$$
\Phi(s, \pi)=\varepsilon(s, \pi) \Phi(1-s, \tilde{\pi}),
$$

where the automorphic irreducible cuspidal representation $\widetilde{\pi}$ is contragredient to $\pi$, and $\varepsilon(s, \pi)=\tau(\pi) Q_{\pi}^{-s}$. Here $Q_{\pi}>0$ is the conductor of $\pi$ ([JacPSShal1]), $\tau(\pi) \in \mathbb{C}^{\times}, Q_{\tilde{\pi}}=Q_{\pi}$, and $\tau(\pi) \tau(\widetilde{\pi})=Q_{\pi}$.

Denote

$$
a_{\pi}\left(p^{k f_{p}}\right)=f_{p} \sum_{v \mid p} \sum_{1 \leq j \leq m} \alpha_{\pi}^{k}(j, v)
$$

$a_{\pi}\left(p^{k}\right)=0$ if $f_{p} \nmid k$, and $c_{\pi}(n)=\Lambda(n) a_{\pi}(n)$, where $\Lambda(n)=\log p$ if $n=p^{k}$ and zero otherwise. Then $a_{\tilde{\pi}}\left(p^{k}\right)=\bar{a}_{\pi}\left(p^{k}\right)$. Then for Re $s>1$, we have

$$
\frac{L^{\prime}}{L}(s, \pi)=-\sum_{n=1}^{\infty} \frac{\Lambda(n) a_{\pi}(n)}{n^{s}} .
$$

By the bound in (2.1) we have

$$
\left|a_{\pi}\left(p^{k f_{p}}\right)\right| \leq m \ell p^{k f_{p}\left((1 / 2)-1 /\left(\ell m^{2}+1\right)\right)}
$$

for any $\pi_{v}$, ramified or unramified. If $\pi_{v}$ is unramified, we have sharper bounds based on (2.3) and (2.4):

$$
\left|a_{\pi}\left(p^{k f_{p}}\right)\right| \leq m \ell p^{k f_{p} / 9}
$$

for $m=2$, and

$$
\left|a_{\pi}\left(p^{k f_{p}}\right)\right| \leq m \ell p^{k f_{p}\left((1 / 2)-1 /\left(m^{2}+1\right)\right)}
$$

for $m \geq 3$.

We will need an explicit formula for the $L$-functions of the smooth type as in [RudSar]. Let $g_{j}$ be a compactly supported smooth function on $\mathbb{R}$. Define $h_{j}(r)$ and $\kappa(\mathbf{h})$ as in (2.6) and (2.7). Let $\rho=(1 / 2)+i \gamma$ be a nontrivial zero of the $L$-function $L(s, \pi)$. By the same arguments step by step as in [RudSar], we prove that

$$
\begin{aligned}
\sum_{\gamma} h_{j}(\gamma)= & \delta(\pi)\left(h_{j}\left(-\frac{i}{2}\right)+h_{j}\left(\frac{i}{2}\right)\right)+\frac{1}{2 \pi} \int_{\mathbb{R}} h_{j}(r) \Omega_{\pi}(r) d r \\
& -\sum_{n=1}^{\infty}\left(\frac{c_{\pi}(n)}{\sqrt{n}} g_{j}(\log n)+\frac{\bar{c}_{\pi}(n)}{\sqrt{n}} g_{j}(-\log n)\right)
\end{aligned}
$$


where the sum on the left side is taken over nontrivial zeros $\rho=(1 / 2)+i \gamma$ of $L(s, \pi)$, and $\delta(\pi)$ equals 1 if the $L$-function is $\zeta(s)$, and zero otherwise. Here

$$
\begin{aligned}
\Omega_{\pi}(t)= & \log Q_{\pi}+\sum_{v \mid \infty} \sum_{1 \leq j \leq m}\left(\frac{\Gamma_{\mathbb{R}}^{\prime}}{\Gamma_{\mathbb{R}}}\left(\frac{1}{2}+\mu_{\pi}(j, v)+i t\right)\right. \\
& \left.+\frac{\Gamma_{\mathbb{R}}^{\prime}}{\Gamma_{\mathbb{R}}}\left(\frac{1}{2}+\bar{\mu}_{\pi}(j, v)-i t\right)\right) \quad \text { if } E \otimes_{\mathbb{Q}} \mathbb{R}=\bigoplus_{v \mid \infty} \mathbb{R} \\
= & \log Q_{\pi}+\sum_{v \mid \infty} \sum_{1 \leq j \leq m}\left(\frac{\Gamma_{\mathbb{C}}^{\prime}}{\Gamma_{\mathbb{C}}}\left(\frac{1}{2}+\mu_{\pi}(j, v)+i t\right)\right. \\
& \left.+\frac{\Gamma_{\mathbb{C}}^{\prime}}{\Gamma_{\mathbb{C}}}\left(\frac{1}{2}+\bar{\mu}_{\pi}(j, v)-i t\right)\right) \text { if } E \otimes_{\mathbb{Q}} \mathbb{R}=\bigoplus_{v \mid \infty} \mathbb{C} .
\end{aligned}
$$

Denote $L=m \log T$. The explicit formula in (4.4) can be rewritten

$$
\begin{aligned}
& \sum_{\gamma} h_{j}\left(\frac{\gamma}{T}\right) e^{-i L \gamma \xi_{j}} \\
= & \delta(\pi)\left(h_{j}\left(-\frac{i}{2 T}\right) T^{-m \xi_{j} / 2}+h_{j}\left(\frac{i}{2 T}\right) T^{m \xi_{j} / 2}\right) \\
& +T g_{j T}\left(T L \xi_{j}\right)+T S_{j}^{+}\left(\xi_{j}\right)+T S_{j}^{-}\left(\xi_{j}\right)
\end{aligned}
$$

where

$$
\begin{aligned}
& g_{j T}(x)=\frac{1}{2 \pi} \int_{\mathbb{R}} h_{j}(r) \Omega_{\pi}(r T) e^{-i r x} d r, \\
& S_{j}^{+}\left(\xi_{j}\right)=-\sum_{n_{j}=1}^{\infty} \frac{\Lambda\left(n_{j}\right) a_{\pi}\left(n_{j}\right)}{\sqrt{n_{j}}} g_{j}\left(T\left(L \xi_{j}+\log n_{j}\right)\right), \\
& S_{j}^{-}\left(\xi_{j}\right)=-\sum_{n_{j}=1}^{\infty} \frac{\Lambda\left(n_{j}\right) \bar{a}_{\pi}\left(n_{j}\right)}{\sqrt{n_{j}}} g_{j}\left(T\left(L \xi_{j}-\log n_{j}\right)\right) .
\end{aligned}
$$

In the following, we will not consider the term with $\delta(\pi)$ on the right side of (4.6), as it is non-zero only for the Riemann zeta function $\zeta(s)$.

5. Rankin-Selberg $L$-functions. For two irreducible automorphic cuspidal representations $\pi$ and $\pi^{\prime}$ of $G L_{m}\left(E_{\mathbb{A}}\right)$, their Rankin-Selberg $L$-function is given by

$$
L\left(s, \pi \times \tilde{\pi}^{\prime}\right)=\prod_{v<\infty} L_{v}\left(s, \pi \times \tilde{\pi}^{\prime}\right)
$$

for $\operatorname{Re} s>1$, where the local factor is

$$
L_{v}\left(s, \pi \times \tilde{\pi}^{\prime}\right)=\prod_{1 \leq j_{1}, j_{2} \leq m}\left(1-\alpha_{\pi}\left(j_{1}, v\right) \bar{\alpha}_{\pi^{\prime}}\left(j_{2}, v\right) q_{E_{v}}^{-s}\right)^{-1} .
$$


Denote

$$
\begin{aligned}
L_{p}\left(s, \pi \times \tilde{\pi}^{\prime}\right) & =\prod_{v \mid p} L_{v}\left(s, \pi \times \tilde{\pi}^{\prime}\right) \\
& =\prod_{v \mid p} \prod_{1 \leq j_{1}, j_{2} \leq m}\left(1-\alpha_{\pi}\left(j_{1}, v\right) \bar{\alpha}_{\pi^{\prime}}\left(j_{2}, v\right) p^{-f_{p} s}\right)^{-1} .
\end{aligned}
$$

Then for Re $s>1$,

$$
\begin{aligned}
& \frac{L^{\prime}}{L}\left(s, \pi \times \tilde{\pi}^{\prime}\right) \\
= & -\sum_{v} \sum_{1 \leq j_{1}, j_{2} \leq m} \sum_{k \geq 1} \frac{f_{p} \log p}{p^{k f_{p} s}} \alpha_{\pi}^{k}\left(j_{1}, v\right) \bar{\alpha}_{\pi^{\prime}}^{k}\left(j_{2}, v\right) \\
= & -\sum_{n \geq 1} \frac{\Lambda(n) a_{\pi \times \tilde{\pi}^{\prime}}(n)}{n^{s}}
\end{aligned}
$$

where

$$
\begin{aligned}
a_{\pi \times \tilde{\pi}^{\prime}}\left(p^{k f_{p}}\right) & =\sum_{v \mid p} f_{p} \sum_{1 \leq j_{1}, j_{2} \leq m} \alpha_{\pi}^{k}\left(j_{1}, v\right) \bar{\alpha}_{\pi^{\prime}}^{k}\left(j_{2}, v\right) \\
& =\sum_{v \mid p} f_{p}\left(\sum_{1 \leq j_{1} \leq m} \alpha_{\pi}^{k}\left(j_{1}, v\right)\right)\left(\sum_{1 \leq j_{2} \leq m} \bar{\alpha}_{\pi^{\prime}}^{k}\left(j_{2}, v\right)\right)
\end{aligned}
$$

and $a_{\pi \times \tilde{\pi}^{\prime}}\left(p^{k}\right)=0$ if $f_{p} \nmid k$. In particular, when $\pi \cong \pi^{\prime}$,

$$
a_{\pi \times \tilde{\pi}}\left(p^{k f_{p}}\right)=\sum_{v \mid p} f_{p}\left|\sum_{1 \leq j \leq m} \alpha_{\pi}^{k}(j, v)\right|^{2} .
$$

Obviously

$$
\left|a_{\pi \times \tilde{\pi}^{\prime}}(n)\right| \leq\left|a_{\pi \times \tilde{\pi}}(n)\right|+\left|a_{\pi^{\prime} \times \tilde{\pi}^{\prime}}(n)\right| .
$$

By the bound for $\left|\alpha_{\pi}(j, v)\right|$ in (2.1), we have

$$
\left|a_{\pi \times \tilde{\pi}}\left(p^{k f_{p}}\right)\right| \leq m^{2} \ell p^{k f_{p}\left(1-2 /\left(\ell m^{2}+1\right)\right)} .
$$

Let $S_{\pi \times \tilde{\pi}^{\prime}}$ be the finite set of primes $p$ such that there is $v \mid p$ with either $\pi_{v}$ or $\pi_{v}^{\prime}$ being ramified. Therefore for any $p \in S_{\pi \times \tilde{\pi}}=S_{\pi}$ we have

$$
\sum_{k \geq 1} \frac{\Lambda\left(p^{k}\right)\left|a_{\pi \times \tilde{\pi}}\left(p^{k}\right)\right|}{p^{k}} \leq m^{2} \ell \log p \sum_{k \geq 1} \frac{1}{p^{2 k /\left(\ell m^{2}+1\right)}}<\infty,
$$


and hence

$$
\sum_{p \in S_{\pi}} \sum_{k \geq 1} \frac{\Lambda\left(p^{k}\right)\left|a_{\pi \times \tilde{\pi}}\left(p^{k}\right)\right|}{p^{k}}<\infty .
$$

On the other hand, absolute convergence of (5.1) for Re $s>1$, together with (5.5), implies that

$$
\sum_{n \leq X} \Lambda(n)\left|a_{\pi \times \tilde{\pi}}(n)\right| \ll_{\varepsilon} X^{1+\varepsilon}
$$

By partial summation, we also have

$$
\sum_{n \leq X}\left|c_{\pi}(n)\right|^{2} \ll_{\varepsilon} X^{1+\varepsilon}
$$

where $c_{\pi}(n)=\Lambda(n) a_{\pi}(n)$ and $a_{\pi}(n)$ is given in (4.1).

Recall that when $\pi^{\prime} \cong \pi \otimes|\operatorname{det}| i \tau_{0}$ for some $\tau_{0} \in \mathbb{R}, L(s, \pi \times \tilde{\pi})$ has simple poles at $s=1+i \tau_{0}$ and $i \tau_{0}$. ([JacPSShal2] and [MoeWal]). Otherwise $L(s, \pi \times \tilde{\pi})$ is entire. Note that the Archimedean part of the Rankin-Selberg $L$-function is

$$
L_{\infty}\left(s, \pi \times \tilde{\pi}^{\prime}\right)=\prod_{v \mid \infty} \prod_{1 \leq j \leq m} \prod_{1 \leq k \leq m^{\prime}} \Gamma_{\mathbb{R}}\left(s+\mu_{\pi \times \tilde{\pi}^{\prime}}(j, k ; v)\right)
$$

or

$$
L_{\infty}\left(s, \pi \times \tilde{\pi}^{\prime}\right)=\prod_{v \mid \infty} \prod_{1 \leq j \leq m} \prod_{1 \leq k \leq m^{\prime}} \Gamma_{\mathbb{C}}\left(s+\mu_{\pi \times \tilde{\pi}^{\prime}}(j, k ; v)\right) .
$$

We will need a trivial bound $\operatorname{Re} \mu_{\pi \times \tilde{\pi}^{\prime}}(j, k ; v)>-1$.

Denote by $\Phi\left(s, \pi \times \tilde{\pi}^{\prime}\right)=L_{\infty}\left(s, \pi \times \tilde{\pi}^{\prime}\right) L\left(s, \pi \times \tilde{\pi}^{\prime}\right)$ the complete RankinSelberg $L$-function. Then by a classical result of Shahidi ([Shah1], [Shah2], [Shah3], and [Shah4]), $\Phi(s, \pi)$ has a functional equation

$$
\Phi\left(s, \pi \times \tilde{\pi}^{\prime}\right)=\varepsilon\left(s, \pi \times \tilde{\pi}^{\prime}\right) \Phi\left(1-s, \tilde{\pi} \times \pi^{\prime}\right),
$$

where $\varepsilon(s, \pi)=\tau\left(\pi \times \tilde{\pi}^{\prime}\right) Q_{\pi \times \tilde{\pi}^{\prime}}^{-s}$. Here $Q_{\pi \times \tilde{\pi}^{\prime}}>0$ is the conductor.

6. Orthogonality. When $\pi$ and $\pi^{\prime}$ are cuspidal representations of $G L_{m}\left(\mathbb{Q}_{\mathbb{A}}\right)$ and $G L_{m^{\prime}}\left(\mathbb{Q}_{\mathbb{A}}\right)$, respectively, with $\pi \neq \pi^{\prime}$, Liu, Wang, and Ye [LiuWangYe] proved the following Selberg orthogonality

$$
\sum_{n \leq x} \frac{(\log n) \Lambda(n) a_{\pi}(n) \bar{a}_{\pi^{\prime}}(n)}{n} \ll \log x,
$$

if at least one of $\pi$ and $\pi^{\prime}$ is self-contragredient. In [LiuWangYe], (6.1) was proved as a consequence of a stronger weighted prime number theorem for a Rankin-Selberg $L$-function, and hence require a zero-free region of the classical type (cf. [Mor1], [Mor2], [GelLapSar], and [Sar]). This is the reason why we have to assume that at least one of $\pi$ and $\pi^{\prime}$ is self-contragredient in (6.1). 
In this section, we will take the approach in [LiuYe2] to get a weighted version of (6.1) for cuspidal representations $\pi$ and $\pi^{\prime}$ over $E$, avoiding the zero-free region and the self-contragredient assumption. Then we will apply an argument of Landau [Land] to remove the weight.

LEMma 6.1. Let $\pi$ and $\pi^{\prime}$ be irreducible automorphic cuspidal representations of $G L_{m}\left(E_{\mathbb{A}}\right)$ and $G L_{m^{\prime}}\left(E_{\mathbb{A}}\right)$ with unitary central characters, respectively. Then

$$
\begin{aligned}
& \sum_{n \leq x}\left(1-\frac{n}{x}\right) \frac{(\log n) \Lambda(n) a_{\pi \times \tilde{\pi}^{\prime}}(n)}{n} \\
= & \frac{1}{2} \log ^{2} x+O(\log x) \quad \text { if } \pi^{\prime} \cong \pi ; \\
= & O(\log x) \quad \text { if } \pi^{\prime} \neq \pi .
\end{aligned}
$$

Proof. The proof follows [LiuYe2] closely, and hence we will only give a brief sketch here and point out the difference. Let $\lambda(s)=\min _{n \leq 0}|s-n|$. Denote by $\mathbb{C}\left(m, m^{\prime}\right)$ the region in the complex plane with the following discs removed:

$$
\left|s-2 n+\mu_{\pi \times \tilde{\pi}^{\prime}}(j, k ; v)\right|<\frac{1}{8 m m^{\prime} \ell}, n \leq 0,1 \leq j \leq m, 1 \leq k \leq m^{\prime}, v \mid \infty,
$$

if $v$ is real, and

$$
\left|s-n+\mu_{\pi \times \tilde{\pi}^{\prime}}(j, k ; v)\right|<\frac{1}{8 m m^{\prime} \ell}, n \leq 0,1 \leq j \leq m, 1 \leq k \leq m^{\prime}, v \mid \infty,
$$

if $v$ is complex. Then for $s \in \mathbb{C}\left(m, m^{\prime}\right)$ and all $j, k$, and $v \mid \infty$,

$$
\lambda\left(\frac{s+\mu_{\pi \times \tilde{\pi}^{\prime}}(j, k ; v)}{2}\right) \geq \frac{1}{16 m m^{\prime} \ell}
$$

if $v$ is real, and

$$
\lambda\left(s+\mu_{\pi \times \tilde{\pi}^{\prime}}(j, k ; v)\right) \geq \frac{1}{16 m m^{\prime} \ell}
$$

if $v$ is complex. Let $\beta(j, k ; v)$ be the fractional part of $\operatorname{Re} \mu_{\pi \times \tilde{\pi}^{\prime}}(j, k ; v)$. In addition we let $\beta(0,0 ; v)=0$ and $\beta(m+1, m+1 ; v)=1$. Then all $\beta(j, k ; v) \in$ $[0,1]$, and hence there exist $\beta\left(j_{1}, k_{1} ; v_{1}\right), \beta\left(j_{2}, k_{2} ; v_{2}\right)$ such that $\beta\left(j_{2}, k_{2} ; v_{2}\right)-$ $\beta\left(j_{1}, k_{1} ; v_{1}\right) \geq 1 /\left(3 m m^{\prime} \ell\right)$ and there is no $\beta(j, k ; v)$ lying between $\beta\left(j_{1}, k_{1} ; v_{1}\right)$ and $\beta\left(j_{2}, k_{2} ; v_{2}\right)$. It follows that the strip

$$
S_{0}=\left\{s \mid \beta\left(j_{1}, k_{1} ; v_{1}\right)+1 /\left(8 m m^{\prime} \ell\right) \leq \operatorname{Re} s \leq \beta\left(j_{2}, k_{2} ; v_{2}\right)-1 /\left(8 m m^{\prime} \ell\right)\right\}
$$

is contained in $\mathbb{C}\left(m, m^{\prime}\right)$. Consequently, for all $n=0,-1,-2, \cdots$, the strips

$$
S_{n}=\left\{s \mid \begin{array}{c}
n+\beta\left(j_{1}, k_{1} ; v_{1}\right)+1 /\left(8 m m^{\prime} \ell\right) \leq \operatorname{Re} s \\
\leq n+\beta\left(j_{2}, k_{2} ; v_{2}\right)-1 /\left(8 m m^{\prime} \ell\right)
\end{array}\right\}
$$

are subsets of $\mathbb{C}\left(m, m^{\prime}\right)$. 
Differentiating (5.1), we get

$$
\left(\frac{L^{\prime}}{L}\right)^{\prime}\left(s, \pi \times \tilde{\pi}^{\prime}\right)=\sum_{n \geq 1} \frac{(\log n) \Lambda(n) a_{\pi \times \tilde{\pi}^{\prime}}(n)}{n^{s}}
$$

for Re $s>1$. By the same proof as in [LiuYe2], §4, using the fact that the RankinSelberg $L$-function is of order one away from its possible poles ([GelShah]), we have the following estimates: For $|T|>2$ there exists $\tau$ with $T \leq \tau \leq T+1$ such that when $-2 \leq \sigma \leq 2$

$$
\left(\frac{L^{\prime}}{L}\right)^{\prime}\left(\sigma+i \tau, \pi \times \tilde{\pi}^{\prime}\right) \ll \log ^{3}\left(Q_{\pi \times \tilde{\pi}^{\prime}}|\tau|\right) ;
$$

If $s$ is in some strip $S_{n}$ as in (6.4) with $n \leq-2$, then

$$
K(s):=\left(\frac{L^{\prime}}{L}\right)^{\prime} L\left(s, \pi \times \tilde{\pi}^{\prime}\right) \ll_{m} 1 .
$$

As in [LiuYe2], §5,

$$
\begin{aligned}
& \sum_{n \leq x}\left(1-\frac{n}{x}\right) \frac{(\log n) \Lambda(n) a_{\pi \times \tilde{\pi}^{\prime}}(n)}{n} \\
= & \frac{1}{2 \pi i} \int_{(1)} K(s+1) \frac{x^{s}}{s(s+1)} d s=\frac{1}{2 \pi i}\left(\int_{1-i T}^{1+i T}+\int_{1-i \infty}^{1-i T} \int_{1+i T}^{1+i \infty}\right) .
\end{aligned}
$$

The last two integrals are bounded by $\ll \int_{T}^{\infty}\left(x / t^{2}\right) d t \ll x / T$. To compute the first integral on the right side of (6.7), we choose $\sigma_{0}$ with $-2<\sigma_{0}<-1$ such that the line $\operatorname{Re}(s)=\sigma_{0}$ is contained in the strip $S_{-2} \subset \mathbb{C}\left(m, m^{\prime}\right)$. Let $T \sim x$ be the $\tau$ such that (6.5) holds. Now we consider the contour

$$
\begin{array}{ll}
C_{1}: & 1 \geq \sigma \geq \sigma_{0}, \quad t=-T ; \\
C_{2}: & \sigma=\sigma_{0}, \quad-T \leq t \leq T ; \\
C_{3}: & \sigma_{0} \leq \sigma \leq 1, \quad t=T .
\end{array}
$$

Note that the two possible poles, some trivial zeros, and certain nontrivial zeros of $L\left(s+1, \pi \times \tilde{\pi}^{\prime}\right)$, as well as $s=0,-1$ are passed by the shifting of the contour. The trivial zeros can be determined by the functional equation (5.7): $s=-1-$ $\mu_{\pi \times \tilde{\pi}^{\prime}}(j, k ; v)$. The trivial bound $\operatorname{Re} \mu_{\pi \times \tilde{\pi}^{\prime}}(j, k ; v)>-1$ will give us $O(\log x)$ for the size the residues from trivial zeros. Integrals on $C_{1}$ and $C_{3}$ are bounded by $O\left(x \log ^{3}\left(Q_{\pi \times \tilde{\pi}^{\prime}} T\right) / T^{2}\right.$ using (6.5), while the integral on $C_{2}$ is bounded by $O(1 / x)$ using (6.6). The residues at $s=0,-1$ will contribute $K(1)+K(0) / x$. The two possible poles are $i \tau_{0}$ and $i \tau_{0}-1$ which can only happen when $m=m^{\prime}$ and $\pi^{\prime} \cong \pi \otimes|\operatorname{det}|^{i \tau_{0}}$ for some $\tau_{0} \in \mathbb{R}$. If $\tau_{0} \neq 0$, these are double poles and they will contribute $O(\log x)$ to (6.7). If $\tau_{0}=0$, i.e., if $\pi \cong \pi^{\prime}$, then $s=0$ becomes a triple pole. Its residue contributes $(1 / 2) \log ^{2} x+O(\log x)$ to $(6.7)$. By the fact of $L\left(s, \pi \times \tilde{\pi}^{\prime}\right) \neq 0$ for $\operatorname{Re} s \geq 1$ ([Shah1]), the contribution from nontrivial zeros is also $O(\log x)$. This completes the proof of Lemma 6.1. 
LEMma 6.2. Let $\pi$ be an irreducible automorphic cuspidal representations of $G L_{m}\left(E_{\mathbb{A}}\right)$ with unitary central characters. Then

$$
\sum_{n \leq x} \frac{(\log n) \Lambda(n) a_{\pi \times \tilde{\pi}}(n)}{n}=\frac{1}{2} \log ^{2} x+O(\log x) .
$$

Proof. Note that this is the case when $\pi \cong \pi^{\prime}$, and hence by (5.3) the series on the left side of (6.2) and (6.8) are of nonnegative terms. By the proof in [RudSar], p.282, we can remove the weight $1-n / x$ from (6.2) when $\pi \cong \pi^{\prime}$.

LEMMA 6.3. Let $\pi$ be an irreducible automorphic cuspidal representations of $G L_{m}\left(E_{\mathbb{A}}\right)$ with unitary central characters. Then

$$
\sum_{n \leq x}(\log n) \Lambda(n) a_{\pi \times \tilde{\pi}}(n) \ll x \log x
$$

Proof. This is deduced from (6.8) by a standard argument of partial summation.

We remark that under the Generalized Ramanujan conjecture, an asymptotic formula was proved in [LiuYe3] for cuspidal self-contragredient representation of $G L_{m}$ over $\mathbb{Q}$ :

$$
\sum_{n \leq x}(\log n) \Lambda(n) a_{\pi \times \tilde{\pi}}(n)=x \log x-x+O(x \exp (-c \sqrt{\log x}))
$$

for a positive constant $c$. The upper bound in (6.9) is nevertheless unconditional and valid for cuspidal representations not self-contragredient.

THEOREM 6.4. Let $\pi$ and $\pi^{\prime}$ be irreducible automorphic cuspidal representations of $G L_{m}\left(E_{\mathbb{A}}\right)$ and $G L_{m^{\prime}}\left(E_{\mathbb{A}}\right)$ with unitary central characters, respectively, such that $\pi^{\prime} \neq \pi$. Then

$$
\sum_{n \leq x} \frac{(\log n) \Lambda(n) a_{\pi \times \tilde{\pi}^{\prime}}(n)}{n} \ll \log x .
$$

Proof. The removal of the weight $1-n / x$ from (6.3) is by an argument of Landau [Land] using (6.9). The proof is given in [LiuWangYe]. To make the present paper self-contained, we reproduce the proof in [LiuWangYe] below.

Denote $c(n)=(\log n) \Lambda(n) a_{\pi \times \tilde{\pi}^{\prime}}(n) / n$, and

$$
C(x)=\sum_{n \leq x} c(n), \quad D(x)=\sum_{n \leq x}\left(1-\frac{n}{x}\right) c(n) .
$$


Then

$$
\int_{0}^{x} C(t) d t=\sum_{n \leq x}(x-n) c(n)=x D(x) .
$$

We begin with an observation that

$$
\begin{aligned}
\int_{x}^{x+v} C(t) d t & =(x+v) D(x+v)-x D(x) \\
& =v D(x+v)+x(D(x+v)-D(v)),
\end{aligned}
$$

where $v=\sqrt{x}$.

By (6.3), we have

$$
v D(x+v) \ll v \log x
$$

as $\pi^{\prime} \neq \pi$. The last term in (6.11) is

$$
\begin{aligned}
& x(D(x+v)-D(v)) \\
= & x \sum_{n \leq x}\left(\frac{n}{x}-\frac{n}{x+v}\right) c(n)+x \sum_{x<n \leq x+v}\left(1-\frac{n}{x+v}\right) c(n) \\
= & : E_{1}+E_{2},
\end{aligned}
$$

say. By (5.4) and Lemma 6.3 we have

$$
\begin{aligned}
\left|E_{1}\right| & \leq \frac{v}{x+v} \sum_{n \leq x} n|c(n)| \\
& \ll \frac{v}{x+v} \sum_{n \leq x}(\log n) \Lambda(n)\left(\left|a_{\pi \times \tilde{\pi}}(n)\right|+\left|a_{\pi^{\prime} \times \tilde{\pi}^{\prime}}(n)\right|\right) \\
& \ll v \log x
\end{aligned}
$$

and

$$
\begin{aligned}
\left|E_{2}\right| & \leq \frac{x v}{x+v} \sum_{x<n \leq x+v}|c(n)| \ll \frac{v}{x+v} \sum_{x<n \leq x+v} n|c(n)| \\
& \ll \frac{v}{x+v} \sum_{x<n \leq x+v}(\log n) \Lambda(n)\left(\left|a_{\pi \times \tilde{\pi}}(n)\right|+\left|a_{\pi^{\prime} \times \tilde{\pi}^{\prime}}(n)\right|\right) \\
& \ll v \log x .
\end{aligned}
$$

Putting (6.12) through (6.14)) into (6.11), we get

$$
\int_{x}^{x+v} C(t) d t \ll v \log x .
$$


Now consider the difference

$$
\begin{aligned}
& \quad\left|\int_{x}^{x+v} C(t) d t-v C(x)\right|=\left|\int_{x}^{x+v}(C(t)-C(x)) d t\right| \\
& \ll v \sum_{x<n \leq x+v}|c(n)| \ll \frac{v}{x} \sum_{x<n \leq x+v} n|c(n)| \\
& \ll \frac{v}{x} \sum_{n \leq x}(\log n) \Lambda(n)\left(\left|a_{\pi \times \tilde{\pi}}(n)\right|+\left|a_{\pi^{\prime} \times \tilde{\pi}^{\prime}}(n)\right|\right) \ll v \log x,
\end{aligned}
$$

by the same argument as above. The desired result (6.10) now follows from this and (6.15).

Under the generalized Ramanujan conjecture, a much precise version of (6.8) and (6.10) was proved in [LiuYe3] for self-contragredient cuspidal representations over $\mathbb{Q}$ :

$$
\begin{aligned}
\sum_{n \leq x} \frac{(\log n) \Lambda(n) a_{\pi \times \tilde{\pi}^{\prime}}(n)=}{n}= & \frac{1}{2} \log ^{2} x+c_{1}+O\{\exp (-c \sqrt{\log x})\} \\
& \text { if } \pi^{\prime} \cong \pi ; \\
= & \frac{x^{i \tau_{0}}}{i \tau_{0}} \log x+\frac{x^{i \tau_{0}}-1}{\tau_{0}^{2}}+c_{2}+O\{\exp (-c \sqrt{\log x})\} \\
& \text { if } \pi^{\prime} \cong \pi \otimes \alpha^{i \tau_{0}} \text { for some } \tau_{0} \in \mathbb{R}^{\times} \\
= & c_{3}+O\{\exp (-c \sqrt{\log x})\} \\
& \text { if } \pi^{\prime} \not \pi \otimes \alpha^{i t} \text { for any } t \in \mathbb{R},
\end{aligned}
$$

where $c, c_{1}, \ldots, c_{3}$ are positive constants.

7. Orthogonality over primes. In this section we will rewrite Lemmas 6.2 and 6.4 as orthogonality taking over primes using Conjecture 2.1 and Hypothesis H. First, according to (6.8) we have

$$
\sum_{\substack{p, k \\ p^{k f_{p}} \leq x}} \frac{k \log ^{2} p}{p^{k f_{p}}} \sum_{v_{i} \mid p} f_{p}^{2}\left|\sum_{1 \leq j \leq m} \alpha_{\pi}^{k}\left(j, v_{i}\right)\right|^{2}=\frac{1}{2} \log ^{2} x+O(\log x) .
$$

Using the bound for $\alpha_{\pi}\left(j, v_{i}\right)$ in $(2.1)$, we have

$$
\begin{aligned}
& \sum_{k>\left(\ell m^{2}+1\right) / 2} \sum_{p} \frac{k \log ^{2} p}{p^{k f_{p}}} \sum_{v \mid p} f_{p}^{2}\left|\sum_{1 \leq j \leq m} \alpha_{\pi}^{k}(j, v)\right|^{2} \\
\ll & \sum_{k>\left(\ell m^{2}+1\right) / 2} \sum_{p} \frac{k \log ^{2} p}{p^{2 k /\left(\ell m^{2}+1\right)}}<\infty .
\end{aligned}
$$


Therefore Hypothesis H implies

$$
\sum_{k \geq 2} \sum_{p} \frac{k \log ^{2} p}{p^{k f_{p}}} \sum_{v \mid p} f_{p}^{2}\left|\sum_{1 \leq j \leq m} \alpha_{\pi}^{k}(j, v)\right|^{2}<\infty .
$$

From (7.1) and (7.2) we conclude that

$$
\sum_{p \leq x} \frac{\log ^{2} p}{p^{f_{p}}} \sum_{v \mid p} f_{p}^{2}\left|\sum_{1 \leq j \leq m} \alpha_{\pi}(j, v)\right|^{2}=\frac{1}{2} \log ^{2} x+O(\log x) .
$$

What we will show below is that the outer sum in (7.3) can be taken over all primes $p \leq x$ which split completely in $E$.

LEMMA 7.1. Let $\pi$ be an automorphic irreducible cuspidal representation $\pi$ of $G L_{m}\left(E_{\mathbb{A}}\right)$ with unitary central character. Assume Hypothesis $H$ over $E$ when $m \geq 5$. Assume Conjecture 2.1 when $m \geq 3$ and there is $p \mid \ell$ such that $p \leq\left(m^{2}+1\right) / 2$. Then

$$
\sum_{\substack{p \leq x \\ \text { splits completely }}} \frac{\log ^{2} p}{p} \sum_{v \mid p}\left|\sum_{1 \leq j \leq m} \alpha_{\pi}(j, v)\right|^{2}=\frac{1}{2} \log ^{2} x+O(\log x) .
$$

Proof. We know that there are only finitely many $p$ with its order of ramification $e_{p}>1$. Thus we can ignore these primes in (7.3) and consider only unramified primes $p$ with $f_{p}=\ell_{p}$. If such a prime $p$ does not split completely in $E$, then $f_{p} \geq 2$. Under Conjecture 2.1, we then have

$$
\begin{aligned}
& \sum_{\substack{p \text { unramified, } \\
\text { not split completely }}} \frac{\log ^{2} p}{p^{f_{p}}} \sum_{v \mid p} f_{p}^{2}\left|\sum_{1 \leq j \leq m} \alpha_{\pi}(j, v)\right|^{2} \\
\ll & \sum_{\substack{p \text { unramified, } \\
\text { not split completely }}} \frac{\log ^{2} p}{p^{f_{p}}} p^{2 f_{p}\left(1 / 2-1 /\left(2 f_{p}\right)-\varepsilon\right)} \\
\ll & \sum_{p} \frac{\log ^{2} p}{p^{1+2 \varepsilon f_{p}}} \ll \sum_{p} \frac{\log ^{2} p}{p^{1+2 \varepsilon}}<\infty .
\end{aligned}
$$

Consequently (7.4) holds.

As in [RudSar], p.300, we can apply partial summation to (7.4) and prove an asymptotic formula for a weighted sum. 
Lemma 7.2. Let $\phi(v)$ be a $C^{1}$ function supported in $|v| \leq(1-\delta) / m$ for some positive $\delta$. Let $\pi$ be an irreducible automorphic cuspidal representations of $G L_{m}\left(E_{\mathbb{A}}\right)$ with unitary central characters. Assume Hypothesis $H$ over $E$ when $m \geq 5$. Assume Conjecture 2.1 when $m \geq 3$ and there is $p \mid \ell$ such that $p \leq$ $\left(m^{2}+1\right) / 2$. Then we have

$$
\begin{aligned}
& \sum_{\substack{p \leq x \\
\text { splits completely }}} \frac{\log ^{2} p}{p} \phi\left(\frac{\log p}{m \log x}\right) \sum_{v_{i} \mid p}\left|\sum_{1 \leq j \leq m} \alpha_{\pi}\left(j, v_{i}\right)\right|^{2} \\
= & m^{2}(\log x)^{2} \int_{0}^{1 / m} v \phi(v) d v+O(\log x),
\end{aligned}
$$

where the implied constant depends on $m$ and $\delta$.

Now let us turn to the case of $\pi \neq \pi^{\prime}$. By Cauchy's inequality, we may remove terms on prime powers from the left side of (6.10). Using the same proof as for Lemma 7.1, we can further remove terms on those primes which do not split completely in $E$.

LEMMA 7.3. Let $\pi$ and $\pi^{\prime}$ be irreducible automorphic cuspidal representations of $G L_{m}\left(E_{\mathbb{A}}\right)$ and $G L_{m^{\prime}}\left(E_{\mathbb{A}}\right)$ with unitary central characters, respectively, such that $\pi^{\prime} \nsucceq \pi$. Assume Hypothesis $H$ over $E$ when $m \geq 5$ or $m^{\prime} \geq 5$. Also assume Conjecture 2.1 when $m \geq 3$ and there is $p \mid \ell$ such that $p \leq\left(m^{2}+1\right) / 2$, or when $m^{\prime} \geq 3$ and there is $p \mid \ell$ such that $p \leq\left(m^{\prime 2}+1\right) / 2$. Then

$$
\begin{aligned}
& \sum_{\substack{p \leq x \\
\text { splits completely }}} \frac{\log ^{2} p}{p} \sum_{v_{i} \mid p}\left(\sum_{1 \leq j_{1} \leq m} \alpha_{\pi}\left(j_{1}, v_{i}\right)\right)\left(\sum_{1 \leq j_{2} \leq m^{\prime}} \bar{\alpha}_{\pi}\left(j_{2}, v_{i}\right)\right) \\
& \ll \log x .
\end{aligned}
$$

Partial summation now can be applied to (7.5) and get the following weighted sum, as in the proof of Proposition 4.5 in [LiuYe1], p.436.

Lemma 7.4. Let $\phi(v)$ be a $C^{1}$ function supported in $|v| \leq(1-\delta) / m$ for some positive $\delta$. Let $\pi$ and $\pi^{\prime}$ be irreducible automorphic cuspidal representations of $G L_{m}\left(E_{\mathbb{A}}\right)$ and $G L_{m^{\prime}}\left(E_{\mathbb{A}}\right)$ with unitary central characters, respectively, such that $\pi^{\prime} \neq \pi$. Then under the same assumption as in Lemma \%.3, we have

$$
\begin{aligned}
& \sum_{\substack{p \leq x \\
\text { splits completely }}} \frac{\log ^{2} p}{p} \phi\left(\frac{\log p}{m \log x}\right) \sum_{v_{i} \mid p}\left(\sum_{1 \leq j_{1} \leq m} \alpha_{\pi}\left(j_{1}, v_{i}\right)\right)\left(\sum_{1 \leq j_{2} \leq m} \bar{\alpha}_{\pi^{\prime}}\left(j_{2}, v_{i}\right)\right) \\
& \ll(\log x) \int_{0}^{1 / m}\left(|\phi(v)|+\left|\phi^{\prime}(v)\right|\right) d v,
\end{aligned}
$$


where the implied constant depends on $m$ and $\delta$.

8. The $n$-level correlation. Let $\Phi \in C^{1}\left(\mathbb{R}^{n}\right)$ be supported in the region $\sum_{1 \leq j \leq n}\left|\xi_{j}\right|<2 / m$. Define $f(x)$ as in (2.8). We will compute the left side of $(3.1)$ :

$$
C_{n}(f, \mathbf{h}, T)=\sum_{\gamma_{1}, \cdots, \gamma_{n}} h_{1}\left(\frac{\gamma_{1}}{T}\right) \cdots h_{n}\left(\frac{\gamma_{n}}{T}\right) f\left(\frac{L}{2 \pi} \gamma_{1}, \ldots, \frac{L}{2 \pi} \gamma_{n}\right)
$$

where $L=m \log T$ and the sum over each $\gamma_{j}$ is taken over nontrivial zeros $\rho_{j}=(1 / 2)+i \gamma_{j}$ of $L(s, \pi)$. Now we use the Fourier transform and get

$$
\begin{aligned}
C_{n}(f, \mathbf{h}, T)= & \int_{\mathbb{R}^{n}} \prod_{j=1}^{n}\left(\sum_{\gamma_{j}} h_{j}\left(\frac{\gamma_{j}}{T}\right) e^{-i L \gamma_{j} \xi_{j}}\right) \Phi(\xi) \delta\left(\xi_{1}+\cdots+\xi_{n}\right) d \xi \\
= & T^{n} \int_{\mathbb{R}^{n}} \prod_{j=1}^{n}\left(g_{j T}\left(T L \xi_{j}\right)+S_{j}^{+}\left(\xi_{j}\right)+S_{j}^{-}\left(\xi_{j}\right)\right) \\
& \times \Phi(\xi) \delta\left(\xi_{1}+\cdots+\xi_{n}\right) d \xi .
\end{aligned}
$$

To compute the product, we set $i_{\mu}=0$ or \pm 1 for $\mu=1, \ldots, n$, and use $i_{\mu}$ to indicate which one of $g_{\mu T}, S_{\mu}^{+}, S_{\mu}^{-}$appears in the term:

$$
\begin{aligned}
C_{n}(f, \mathbf{h}, T)= & T^{n} \sum_{-1 \leq i_{1}, \ldots, i_{n} \leq 1} \int_{\mathbb{R}^{n}} \prod_{i_{\mu}=0} g_{\mu T}\left(T L \xi_{\mu}\right) \prod_{i_{\mu}=1} S_{\mu}^{+}\left(\xi_{\mu}\right) \prod_{i_{\mu}=-1} S_{\mu}^{-}\left(\xi_{\mu}\right) \\
& \times \Phi(\xi) \delta\left(\xi_{1}+\cdots+\xi_{n}\right) d \xi .
\end{aligned}
$$

Now we use (4.7) and (4.8) to expand $S_{\mu}^{ \pm}\left(\xi_{\mu}\right)$. Recall that $\Lambda\left(n_{\mu}\right) a_{\pi}\left(n_{\mu}\right)=c_{\pi}\left(n_{\mu}\right)$. We have

$$
C_{n}(f, \mathbf{h}, T)=\sum_{-1 \leq i_{1}, \ldots, i_{n} \leq 1}(-1)^{i_{1}+\cdots+i_{n}} C_{i_{1} \cdots i_{n}}(T)
$$

and

$$
C_{i_{1} \cdots i_{n}}(T)=\sum_{\substack{n_{\mu} \geq 1 \\ \text { for } i_{\mu} \neq 0}}\left(\prod_{i_{\mu}=1} \frac{c_{\pi}\left(n_{\mu}\right)}{n_{\mu}^{1 / 2}}\right)\left(\prod_{i_{\mu}=-1} \frac{\overline{c_{\pi}}\left(n_{\mu}\right)}{n_{\mu}^{1 / 2}}\right) A_{i_{1} \cdots i_{n}}(\mathbf{n}, T) .
$$

Here $\mathbf{n}=\left(n_{1}, \cdots, n_{n}\right)$ where $n_{\mu}=1$ if $i_{\mu}=0$, and

$$
\begin{aligned}
& A_{i_{1} \cdots i_{n}}(\mathbf{n}, T) \\
= & T^{n} \int_{\mathbb{R}^{n}} \prod_{i_{\mu}=0} g_{\mu T}\left(T L \xi_{\mu}\right) \prod_{i_{\mu} \neq 0} g_{\mu}\left(T\left(L \xi_{\mu}+i_{\mu} \log n_{\mu}\right)\right) \\
& \times \Phi(\xi) \delta\left(\xi_{1}+\cdots+\xi_{n}\right) d \xi .
\end{aligned}
$$


We will follow [RudSar] and [LiuYe1] closely to estimate $C_{n}(f, \mathbf{h}, T)$ and point out differences between the situation at hand and those discussed there. First, in the definition of $\Omega_{\pi}(r)$ and hence of $g_{T}(x)$, there are sums of $\Gamma_{\mathbb{C}}^{\prime} / \Gamma_{\mathbb{C}}$ or possibly more terms of $\Gamma_{\mathbb{R}}^{\prime} / \Gamma_{\mathbb{R}}$. These will cause no problem, since either over $\mathbb{C}$ or over $\mathbb{R}$, Re $\mu_{\pi}(j, v)>-1 / 2$ by [RudSar]. By Stirling's formula, we thus have

$$
\frac{\Gamma_{\mathbb{C}}^{\prime}}{\Gamma_{\mathbb{C}}}(s)=2 \frac{\Gamma_{\mathbb{R}}^{\prime}}{\Gamma_{\mathbb{R}}}(s)+O\left(\frac{1}{s}\right) .
$$

Therefore, Lemma 4.1 in [RudSar] is still true here. That is,

$$
\begin{aligned}
g_{T}(x) & \ll \frac{e^{-\sigma x / T}}{x}\left(1-e^{-\sigma x / 2}\right)+e^{-\sigma x} \log T \\
g_{T}(x) & \ll \begin{cases}\log T & \text { if }|x| \ll \log \log T, \\
1 /|x| & \text { if }|x| \gg \log \log T,\end{cases} \\
\int_{\mathbb{R}}\left|g_{T}(x)\right| d x & \ll \log T .
\end{aligned}
$$

Consequently, the integral in (8.2) converges absolutely.

We want to prove Proposition 4.1 of [RudSar] in our case. That is, we will show that

$$
C_{i_{1}, \ldots, i_{n}}(T)=\widetilde{C}_{i_{1}, \ldots, i_{n}}(T)+O\left(T^{1-\delta / 3}\right),
$$

if $\Phi(\xi)$ in (2.8) is supported in $\left|\xi_{1}\right|+\cdots+\left|\xi_{n}\right| \leq(2-\delta) / m$, where

$$
\tilde{C}_{i_{1} \cdots i_{n}}(T)=\sum_{\substack{1 \leq n_{\mu} \ll T \text { for } i_{\mu} \neq 0, M N \ll T^{2-\delta} \\ M=N}}\left(\prod_{i_{\mu}=1} \frac{c_{\pi}\left(n_{\mu}\right)}{n_{\mu}^{1 / 2}}\right)\left(\prod_{i_{\mu}=-1} \frac{\overline{c_{\pi}}\left(n_{\mu}\right)}{n_{\mu}^{1 / 2}}\right) \tilde{A}_{i_{1} \cdots i_{n}}(\mathbf{n}, T)
$$

and

$$
\begin{aligned}
& \tilde{A}_{i_{1} \cdots i_{n}}(\mathbf{n}, T) \\
= & T_{\substack{\mathbb{R}^{n} \\
\left|T L \xi_{\mu}\right| \ll T^{\delta / 3} \\
\text { for } i_{\mu}=0}} \prod_{i_{\mu}=0} g_{\mu T}\left(T L \xi_{\mu}\right) \\
& \times \prod_{i_{\mu} \neq 0} g_{\mu}\left(T\left(L \xi_{\mu}+i_{\mu} \log n_{\mu}\right)\right) \Phi(\xi) \delta\left(\xi_{1}+\cdots+\xi_{n}\right) d \xi .
\end{aligned}
$$

To do this, we first point out that Lemmas 4.2 and 4.3 of [RudSar], i.e., Lemmas 7.1 and 7.2 of [LiuYe1], hold in the present case. 
Lemma 8.1. $\quad A_{i_{1} \cdots i_{n}}(\mathbf{n}, T)=0$ unless $\left|n_{\mu}\right| \ll T$ for $i_{\mu} \neq 0$ and $\prod_{i_{\mu} \neq 0} n_{\mu} \ll$ $T^{2-\delta}$.

Lemma 8.2. $\quad \tilde{A}_{i_{1} \cdots i_{n}}(\mathbf{n}, T)=0$ unless $\left|n_{\mu}\right| \ll T$ for $i_{\mu} \neq 0, \prod_{i_{\mu} \neq 0} n_{\mu} \ll$ $T^{2-\delta}$, and $\prod_{i_{\mu}=1} n_{\mu}=\prod_{i_{\mu}=-1} n_{\mu}$.

Lemma 4.4 of [RudSar] then holds in our case, as it is based on the bounds for $g_{T}$ and Lemmas 8.1 and 8.2 above.

LEMMA 8.3. If $M N \ll T^{2-\delta}$, then

$$
A_{i_{1} \cdots i_{n}}(\mathbf{n}, T)-\tilde{A}_{i_{1} \cdots i_{n}}(\mathbf{n}, T) \ll \begin{cases}T^{1-\delta / 3} L^{-r-s} & \text { if }\left|\log \frac{M}{N}\right| \ll T^{\delta / 3-1}, \\ \frac{L^{-r-s}}{|\log M / N|} & \text { if }\left|\log \frac{M}{N}\right| \gg T^{\delta / 3-1}\end{cases}
$$

where $r=\sum_{i_{\mu}=1} 1$ and $s=\sum_{i_{\mu}=-1} 1$.

Now we can write

where

$$
C_{i_{1} \cdots i_{n}}(T)=\tilde{C}_{i_{1} \cdots i_{n}}(T)+\sum_{\mathrm{diag}}+\sum_{\mathrm{off}}
$$

$$
\begin{aligned}
\sum_{\text {diag }}= & \sum_{\substack{1 \leq n_{\mu} \ll T \text { for } i_{\mu} \neq 0, M N \ll T^{2-\delta},|\log M / N| \ll T^{\delta / 3-1}}}\left(\prod_{i_{\mu}=1} \frac{c_{\pi}\left(n_{\mu}\right)}{n_{\mu}^{1 / 2}}\right)\left(\prod_{i_{\mu}=-1} \frac{\overline{c_{\pi}}\left(n_{\mu}\right)}{n_{\mu}^{1 / 2}}\right) \\
& \times\left(A_{i_{1} \cdots i_{n}}(\mathbf{n}, T)-\tilde{A}_{i_{1} \cdots i_{n}}(\mathbf{n}, T)\right) \\
\ll & \frac{T^{1-\delta / 3}}{L^{r+s}} \sum_{\substack{M=N, \prod_{\mu} \neq 0}} \prod_{i_{\mu} \neq 0} \frac{\left|c_{\pi}\left(n_{\mu}\right)\right|}{\sqrt{n_{\mu}}},
\end{aligned}
$$

and

$$
\begin{aligned}
\sum_{\mathrm{off}}= & \sum_{\substack{1 \leq n_{\mu} \ll T \text { for } i_{\mu} \neq 0, M N \ll T^{2-\delta},|\log M / N| \gg T^{\delta / 3-1}}}\left(\prod_{i_{\mu}=1} \frac{c_{\pi}\left(n_{\mu}\right)}{n_{\mu}^{1 / 2}}\right)\left(\prod_{i_{\mu}=-1} \frac{\overline{c_{\pi}}\left(n_{\mu}\right)}{n_{\mu}^{1 / 2}}\right) \\
& \times\left(A_{i_{1} \cdots i_{n}}(\mathbf{n}, T)-\tilde{A}_{i_{1} \cdots i_{n}}(\mathbf{n}, T)\right) \\
\ll & \frac{1}{L^{r+s}} \sum_{\substack{M N \ll T^{2-\delta}, M \neq N}} \frac{1}{\sqrt{M N}|\log M / N|} \\
& \times \sum_{\prod_{i_{\mu}=1} n_{\mu}=M} \prod_{i_{\mu}=1}\left|c_{\pi}\left(n_{\mu}\right)\right| \sum_{\prod_{i_{\mu}=-1} n_{\mu}=N} \prod_{i_{\mu}=-1}\left|c_{\pi}\left(n_{\mu}\right)\right|
\end{aligned}
$$


by Lemma 8.3. We will prove later that

$$
\sum_{\substack{M=N, \prod_{i \mu \neq 0} n_{\mu} \ll T^{2-\delta}}} \prod_{i_{\mu} \neq 0} \frac{\left|c_{\pi}\left(n_{\mu}\right)\right|}{n_{\mu}^{1 / 2}} \ll(\log T)^{r+s}
$$

from which we can deduce $\sum_{\text {diag }} \ll T^{1-\delta / 3}$. Denote

$$
a_{k}(M)=\sum_{n_{1} \cdots n_{k}=M} \prod_{1 \leq j \leq k}\left|c_{\pi}\left(n_{j}\right)\right|
$$

Then using (5.6) we can still prove Lemma 4.5 of [RudSar]:

$$
\sum_{m \leq X} a_{k}(m)^{2} \ll_{\varepsilon} X^{1+\varepsilon}
$$

Using the same arguments as in [RudSar], pp.293-294, we get

$$
\sum_{\text {off }} \ll T^{1+\varepsilon-\delta / 2}
$$

for any $\varepsilon>0$. Therefore, (8.4) is valid after we prove (8.7).

Back to (8.6), we change variables to

$$
y_{\mu}= \begin{cases}T L \xi_{\mu} & \text { if } i_{\mu}=0 \\ T\left(L \xi_{\mu}+i_{\mu} \log n_{\mu}\right) & \text { if } i_{\mu} \neq 0\end{cases}
$$

and get

$$
\begin{aligned}
\tilde{A}_{i_{1} \cdots i_{n}}(\mathbf{n}, T)= & \frac{T}{L^{n-1}} \int_{V} \prod_{i_{\mu}=0} g_{\mu T}\left(y_{\mu}\right) \prod_{i_{\mu} \neq 0} g_{\mu}\left(y_{\mu}\right) \\
& \times \Phi\left(\frac{y_{1}}{T L}-\frac{i_{1} \log n_{1}}{L}, \cdots, \frac{y_{n}}{T L}-\frac{i_{n} \log n_{n}}{L}\right) d y \\
+ & \frac{T}{L^{n-1}} \int_{V} \prod_{i_{\mu}=0} g_{\mu T}\left(y_{\mu}\right) \prod_{i_{\mu} \neq 0} g_{\mu}\left(y_{\mu}\right) d y \\
& \times\left(\Phi\left(-\frac{i_{1} \log n_{1}}{L}, \ldots,-\frac{i_{n} \log n_{n}}{L}\right)+O\left(T^{-1+\delta / 3}\right)\right),
\end{aligned}
$$

where $V$ is defined by $\sum_{j} y_{j}=0,\left|y_{\mu}\right| \ll 1$ for $i_{\mu} \neq 0$, and $\left|y_{\mu}\right| \ll T^{\delta / 3}$ for $i_{\mu}=0$. 
By Stirling's approximation formula and (8.3), we get from (4.5) that

$$
\Omega_{\pi}(T r)=m \ell \log (T r)+O(1)
$$

for $r \geq 1$. Consequently

$$
\frac{1}{2 \pi} \int_{\mathbb{R}} h_{1}(r) \cdots h_{n}(r) \Omega_{\pi}(T r)^{k} d r=\frac{\kappa(\mathbf{h})}{2 \pi} L^{k} \ell^{k}+O\left(L^{k-1}\right),
$$

and hence

$$
\int_{V} \prod_{i_{\mu}=0} g_{\mu T}\left(y_{\mu}\right) \prod_{i_{\mu} \neq 0} g_{\mu}\left(y_{\mu}\right) d y=\frac{\kappa(\mathbf{h})}{2 \pi} L^{k} \ell^{k}+O\left(L^{k-1}\right)
$$

where $k=n-r-s$ is the number of $\mu$ with $i_{\mu}=0$. This is Lemma 4.6 of [RudSar]. Back to (8.6), we now have for $r+s>0$ that

$$
\tilde{A}_{i_{1} \cdots i_{n}}(\mathbf{n}, T)=\frac{T \ell^{n-r-s}}{L^{r+s-1}} \frac{\kappa(\mathbf{h})}{2 \pi} \Phi\left(-\frac{i_{1} \log n_{1}}{L}, \ldots,-\frac{i_{n} \log n_{n}}{L}\right)+O\left(\frac{T}{L^{r+s}}\right) .
$$

From (8.4) and (8.5) we thus obtain

$$
\begin{aligned}
C_{i_{1} \cdots i_{n}}(T)= & \frac{\kappa(\mathbf{h})}{2 \pi} \frac{T \ell^{n-r-s}}{L^{r+s-1}}\left(1+O\left(\frac{1}{L}\right)\right) \\
& \times \sum_{\substack{1 \leq n_{\mu} \ll T \text { for } i_{\mu} \neq 0, \prod_{i_{\mu}=1} n_{\mu}=\prod_{i_{\mu}=-1} n_{\mu}}}\left(\prod_{i_{\pi}} \frac{c_{\pi}\left(n_{\mu}\right)}{n_{\mu}^{1 / 2}}\right)\left(\prod_{i_{\mu}=-1} \frac{\overline{c_{\pi}}\left(n_{\mu}\right)}{n_{\mu}^{1 / 2}}\right) \\
& \quad \prod_{i_{\mu} \neq 0} n_{\mu} \ll T^{2} \\
& \times \Phi\left(-\frac{i_{1} \log n_{1}}{L}, \ldots,-\frac{i_{n} \log n_{n}}{L}\right)+O\left(T^{1-\delta / 3}\right)
\end{aligned}
$$

when $i_{1}, \ldots, i_{n}$ are not all zero, and

$$
C_{0 \cdots 0}(T)=\frac{\kappa(\mathbf{h})}{2 \pi} T L \ell^{n} \Phi(0, \ldots, 0)+O(T) .
$$

If we assume Lemma 8.4 below, then (8.8) can be rewritten:

$$
\begin{aligned}
& C_{i_{1} \cdots i_{n}}(T) \\
& =\frac{\kappa(\mathbf{h})}{2 \pi} \frac{T \ell^{n-r-s}}{L^{r+s-1}} \sum_{1 \leq n_{\mu} \ll T \text { for } i_{\mu} \neq 0,}\left(\prod_{i_{\mu}=1} \frac{c_{\pi}\left(n_{\mu}\right)}{n_{\mu}^{1 / 2}}\right) \\
& \prod_{i_{\mu}=1} n_{\mu}=\prod_{i_{\mu}=-1} n_{\mu} \\
& \prod_{i_{\mu} \neq 0} n_{\mu} \ll T^{2} \\
& \times\left(\prod_{i_{\mu}=-1} \frac{\overline{c_{\pi}}\left(n_{\mu}\right)}{n_{\mu}^{1 / 2}}\right) \Phi\left(-\frac{i_{1} \log n_{1}}{L}, \ldots,-\frac{i_{n} \log n_{n}}{L}\right)+O(T)
\end{aligned}
$$

when $r+s>0$. This is indeed Lemma 4.7 of [RudSar]. 
Now let us turn to Lemma 3.8 of [RudSar]. From the bound for $a_{\pi}\left(p^{k}\right)$ in (4.2) we get

$$
\left|c_{\pi}\left(p^{k}\right)\right| \leq m \ell(\log p) p^{k((1 / 2)-\beta)}
$$

with $\beta=1 /\left(\ell m^{2}+1\right)>0$, which is essentially the same bound as in [RudSar] and [LiuYe1]. Consequently for any $K>1 / \beta$ we have (4.54) of [RudSar]:

$$
\sum_{p} \sum_{k_{1}+\cdots+k_{t} \geq K} \frac{\left|c_{\pi}\left(p^{k_{1}}\right) \cdots c_{\pi}\left(p^{k_{t}}\right)\right|}{p^{\left(k_{1}+\cdots+k_{t}\right) / 2}} \ll \sum_{p} \frac{\log ^{t} p}{p^{K \beta}}<\infty
$$

From (5.5) we deduce that

$$
\sum_{p} \frac{\left|c_{\pi}\left(p^{k}\right)\right|^{2}}{p^{k+\delta}}<\infty
$$

for fixed $k$ and $\delta>0$. By an argument in [RudSar], we can prove that

$$
\sum_{p} \sum_{k_{1}+\cdots+k_{t}=s} \frac{\left|c_{\pi}\left(p^{k_{1}}\right) \cdots c_{\pi}\left(p^{k_{t}}\right)\right|}{p^{s / 2}}
$$

is bounded for $s<K$ fixed. Together with (8.11), this implies that Lemma 3.8 of [RudSar] is valid:

$$
\sum_{p} \sum_{\substack{k_{i}, l_{j} \geq 1, k_{1}+\cdots+k_{r}=l_{1}+\cdots+l_{s}}} \frac{\left|c_{\pi}\left(p^{k_{1}}\right) \cdots c_{\pi}\left(p^{k_{r}}\right) \bar{c}_{\pi}\left(p^{l_{1}}\right) \cdots \bar{c}_{\pi}\left(p^{l_{s}}\right)\right|}{p^{k_{1}+\cdots+k_{r}}}<\infty
$$

when $r+s \geq 3$.

Now we prove the following lemma which is Lemma 3.9 of [RudSar].

Lemma 8.4. Assume $m=2$ or Hypothesis $H$. If $1 \leq r \leq s$, then

$$
\sum_{\substack{p_{\mu}^{k_{\mu}} \leq x \text { for } i_{\mu} \neq 0, \prod_{p_{\mu}=1}^{p_{\mu}}=\prod_{i_{\mu}=-1} p_{\mu}^{k_{\mu}}}}\left(\prod_{i_{\mu}=1} \frac{c_{\pi}\left(p_{\mu}^{k_{\mu}}\right)}{p_{\mu}^{k_{\mu} / 2}}\right)\left(\prod_{i_{\mu}=-1} \frac{\overline{c_{\pi}}\left(p_{\mu}^{k_{\mu}}\right)}{p_{\mu}^{k_{\mu} / 2}}\right) \ll \begin{cases}(\log x)^{2 r} & \text { if } r=s, \\ (\log x)^{2 r-2} & \text { if } r<s .\end{cases}
$$


Proof. Using the argument in [RudSar] after (4.59), the lemma is reduced to estimation of

$$
\begin{aligned}
\sum_{\substack{p, k \\
p^{k} \leq x}} \frac{\left|c_{\pi}\left(p^{k}\right)\right|^{2}}{p^{k}}= & \sum_{\substack{p, k \\
p^{k} \leq x}} \frac{\log ^{2} p}{p^{k}} \sum_{\substack{v_{1}, v_{2}\left|p, f_{1}, f_{2}\right| k}} f_{1} f_{2}\left(\sum_{1 \leq j_{1} \leq m} \alpha_{\pi}^{k / f_{1}}\left(j_{1}, v_{1}\right)\right) \\
& \times\left(\sum_{\substack{1 \leq j_{2} \leq m \\
\pi}}^{k / f_{2}}\left(j_{2}, v_{2}\right)\right) \\
\ll & \left(\sum_{\substack{p, k \\
p^{k} \leq x}} \frac{\log ^{2} p}{p^{k}} \sum_{v_{1} \mid p,} f_{1}^{2}\left|\sum_{1 \leq j_{1} \leq m} \alpha_{\pi}^{k / f_{1}}\left(j_{1}, v_{1}\right)\right|^{2}\right)^{1 / 2} \\
& \times\left(\sum_{\substack{p, k \\
f_{1} \mid k}} \frac{\log ^{2} p}{p^{k}} \sum_{v_{2} \mid p,} f_{2}^{2}\left|\sum_{1 \leq j_{2} \leq m} \alpha_{\pi}^{k / f_{2}}\left(j_{2}, v_{2}\right)\right|^{2}\right)^{1 / 2}
\end{aligned}
$$

by Cauchy. Using our results in (7.1) from the Rankin-Selberg $L$-functions, we conclude that

$$
\sum_{\substack{p, k \\ p^{k} \leq x}} \frac{\left|c_{\pi}\left(p^{k}\right)\right|^{2}}{p^{k}} \ll \log ^{2} x .
$$

This proves the Lemma.

Lemma 8.4 implies (8.7) and hence (8.4). It also completed the proof of (8.10). Let $S_{i_{1} \cdots i_{n}}$ be the set of bijective maps from the set $\left\{\mu \mid i_{\mu}=1\right\}$ onto the set $\left\{\mu \mid i_{\mu}=-1\right\}$ when $r=s$. Then (8.10) can be further reduced to

$$
\begin{aligned}
C_{i_{1} \cdots i_{n}}(T)= & \frac{\kappa(\mathbf{h})}{2 \pi} \frac{T L}{L^{2 r}} \sum_{p_{\mu} \leq T \text { for } i_{\mu}=1} \sum_{\sigma \in S_{i_{1} \cdots i_{n}}} \prod_{i_{\mu}=1} \frac{\left|c_{\pi}\left(p_{\mu}\right)\right|^{2}}{p_{\mu}} \\
& \times \Phi\left(z_{1}, \cdots, z_{n}\right)+O(T)
\end{aligned}
$$

where $z_{\mu}=-\left(\log p_{\mu}\right) / L$ and $z_{\sigma(\mu)}=\left(\log p_{\mu}\right) / L$ if $i_{\mu}=1$, and $z_{\mu}=0$ if $i_{\mu}=0$, when $r=s>0$. When $r \neq s, C_{i_{1} \cdots i_{n}}(T)=O(T)$ by Lemma 8.4. Consequently 
we can go back to (8.1) and (8.9) to write

$$
\begin{aligned}
& \sum_{\substack{\gamma_{1}, \cdots, \gamma_{n} \\
T}} h_{1}\left(\frac{\gamma_{1}}{T}\right) \cdots h_{n}\left(\frac{\gamma_{n}}{T}\right) f\left(\frac{L \gamma_{1}}{2 \pi}, \cdots, \frac{L \gamma_{n}}{2 \pi}\right) \\
= & \frac{\kappa(\mathbf{h})}{2 \pi} T L \ell^{n} \Phi(0, \cdots, 0) \\
& +\sum_{-1 \leq i_{1}, \cdots, i_{n} \leq 1} \frac{\kappa(\mathbf{h})}{2 \pi} \frac{T \ell^{n-2 r}}{L^{2 r-1}} \sum_{\sigma \in S_{i_{1} \cdots i_{n}}} \prod_{i_{\mu}=1} \sum_{p_{\mu} \leq T} \frac{\left|c_{\pi}\left(p_{\mu}\right)\right|^{2}}{p_{\mu}} \\
& \times \Phi\left(\frac{\log p_{1}}{L}, \ldots, \frac{\log p_{r}}{L},-\frac{\log p_{1}}{L}, \ldots,-\frac{\log p_{r}}{L}, 0, \ldots, 0\right) \\
& +O(T)
\end{aligned}
$$

where on the left side, for each $j=1, \ldots, n, \rho_{j}=(1 / 2)+i \gamma_{j}$ is taken over all nontrivial zeros of $L(s, \pi)$.

Estimation of (8.12) is thus reduced to asymptotic behavior of

$$
\sum_{p \leq x} \frac{\left|c_{\pi}(p)\right|^{2}}{p} \phi\left(\frac{\log p}{m \log x}\right)
$$

where $\phi(v)$ is a $C^{1}$ function supported in $|v| \leq(1-\delta) / m$ for some positive $\delta$. By the definition of $c_{\pi}(n)$ in (4.1), we get

$$
\begin{aligned}
& \sum_{p \leq x} \frac{\left|c_{\pi}(p)\right|^{2}}{p} \phi\left(\frac{\log p}{m \log x}\right) \\
= & \sum_{p \leq x} \frac{\log ^{2} p}{p} \phi\left(\frac{\log p}{m \log x}\right)\left|\sum_{\substack{v \mid p \\
f_{p}=1}} \sum_{1 \leq j \leq m} \alpha_{\pi}(j, v)\right|^{2} \\
= & \sum_{p \leq x} \frac{\log ^{2} p}{p} \phi\left(\frac{\log p}{m \log x}\right) \\
& \times \sum_{\substack{v_{1}\left|p, v_{2}\right| p \\
f_{p}=1}}\left(\sum_{1 \leq j_{1} \leq m} \alpha_{\pi}\left(j_{1}, v_{1}\right)\right)\left(\sum_{1 \leq j_{2} \leq m} \bar{\alpha}_{\pi}\left(j_{2}, v_{2}\right)\right) .
\end{aligned}
$$

9. The proof of Theorem 3.1. In this section we will assume that $E$ is a Galois (not necessarily cyclic) extension of $\mathbb{Q}$ of degree $\ell$. We plan to compute (8.12) and deduce the $n$-level correlation of normalized nontrivial zeros of $L(s, \pi)$ from (8.12). The estimation (8.12) is based on the asymptotic behavior of (8.13). 
Note that only those primes $p$ with $f_{p}=1$ contribute to (8.13). Consequently, the outermost sum on the right side of (8.13) is actually taken over (i) primes $p \leq x$ which split completely in $E$, and (ii) primes $p \leq x$ above which each local field $E_{v}$ is fully ramified $\left(f_{p}=1\right)$. Note that there are only finitely many primes $p$ which satisfy (ii) but not (i). We can thus rewrite (8.13) as

$$
\begin{aligned}
& \sum_{p \leq x} \frac{\left|c_{\pi}(p)\right|^{2}}{p} \phi\left(\frac{\log p}{m \log x}\right) \\
= & \sum_{\substack{p \leq x \\
\text { splits completely }}} \frac{\log ^{2} p}{p} \phi\left(\frac{\log p}{m \log x}\right) \\
& \times \sum_{v_{1}\left|p, v_{2}\right| p}\left(\sum_{1 \leq j_{1} \leq m} \alpha_{\pi}\left(j_{1}, v_{1}\right)\right)\left(\sum_{1 \leq j_{2} \leq m} \bar{\alpha}_{\pi}\left(j_{2}, v_{2}\right)\right)+O(1) .
\end{aligned}
$$

Now we consider two cases. First let us assume that for any $\sigma \in \mathrm{Gal}_{E / \mathbb{Q}}, \pi$ and $\pi^{\sigma}$ are equivalent. Under this condition, we have $\pi_{v_{1}} \cong \pi_{v_{2}}$ and

$$
\left\{\alpha_{\pi}\left(j_{1}, v_{1}\right) \mid 1 \leq j_{1} \leq m\right\}=\left\{\alpha_{\pi}\left(j_{2}, v_{2}\right) \mid 1 \leq j_{2} \leq m\right\}
$$

for any $v_{1}$ and $v_{2}$ lying above a completely splitting $p$. Consequently (9.1) becomes

$$
\begin{aligned}
& \sum_{p \leq x} \frac{\left|c_{\pi}(p)\right|^{2}}{p} \phi\left(\frac{\log p}{m \log x}\right) \\
= & \ell \sum_{\substack{p \leq x \\
\text { splits completely }}} \frac{\log ^{2} p}{p} \phi\left(\frac{\log p}{m \log x}\right) \sum_{v \mid p}\left|\sum_{1 \leq j \leq m} \alpha_{\pi}\left(j_{1}, v\right)\right|^{2}+O(1) .
\end{aligned}
$$

By Lemma 7.2, we get

$$
\sum_{p \leq T} \frac{\left|c_{\pi}(p)\right|^{2}}{p} \phi\left(\frac{\log p}{L}\right)=\ell m^{2} \log ^{2} T \int_{0}^{1 / m} v \phi(v) d v+O(\log T) .
$$


This implies an asymptotic formula

$$
\begin{aligned}
& \sum_{\gamma_{1}, \cdots, \gamma_{n}} h_{1}\left(\frac{\gamma_{1}}{T}\right) \cdots h_{n}\left(\frac{\gamma_{n}}{T}\right) f\left(\frac{L \gamma_{1}}{2 \pi}, \cdots, \frac{L \gamma_{n}}{2 \pi}\right) \\
= & \frac{\kappa(\mathbf{h})}{2 \pi} T L \ell^{n} \Phi(0, \cdots, 0) \\
& +\frac{\kappa(\mathbf{h})}{2 \pi} T L \sum_{-1 \leq i_{1}, \ldots, i_{n} \leq 1} \ell^{n-r} \sum_{\sigma \in S_{i_{1}} \cdots i_{n}} \int_{0}^{1 / m} \cdots \int_{0}^{1 / m} v_{1} \cdots v_{r} \\
= & \frac{\kappa(\mathbf{h})}{2 \pi} T L \ell^{n} \Phi(0, \cdots, 0) \\
& +\frac{\kappa(\mathbf{h})}{2 \pi} T L \sum_{1 \leq r \leq n / 2} \frac{n ! \ell^{n-r}}{2^{r} r !(n-2 r) !} \int_{\mathbb{R}^{r}}\left|v_{1}\right| \cdots\left|v_{r}\right| \\
& \times \Phi\left(v_{1}, \ldots, v_{r},-v_{1}, \ldots,-v_{r}, 0, \ldots, 0\right) d v_{1} \cdots d v_{r}+O(T) .
\end{aligned}
$$

Next let us consider the case that $\pi$ is not stable under whole $\mathrm{Gal}_{E / \mathbb{Q}}$. Let $\mathrm{G}_{\pi}=\left\{\sigma \in \mathrm{Gal}_{E / \mathbb{Q}} \mid \pi^{\sigma} \cong \pi\right\}$ be the subgroup of $\mathrm{Gal}_{E / \mathbb{Q}}$ fixing $\pi$. Denote by $a$ be the number of elements in $\mathrm{G}_{\pi}$, so that $a \mid \ell$ and $1 \leq a<\ell$. Then according to [Rog], for any two places $v_{1}$ and $v_{2}$ lying above a completely splitting $p, \pi_{v_{1}} \cong \pi_{v_{2}}$ if and only if $E_{v_{1}}$ is mapped to $E_{v_{2}}$ by some $\sigma \in \mathrm{G}_{\pi}$.

Consequently (9.1) becomes

$$
\begin{aligned}
& \sum_{p \leq x} \frac{\left|c_{\pi}(p)\right|^{2}}{p} \phi\left(\frac{\log p}{m \log x}\right) \\
& =\sum_{\substack{\sigma \in \operatorname{Gal}_{E / \mathbb{Q}} \\
\pi \cong \pi^{\sigma}}} \sum_{\substack{p \leq x \\
\text { splits } \\
\text { completely }}} \frac{\log ^{2} p}{p} \phi\left(\frac{\log p}{m \log x}\right) \sum_{v \mid p}\left|\sum_{1 \leq j \leq m} \alpha_{\pi}(j, v)\right|^{2} \\
& +\sum_{\substack{\sigma \in \mathrm{Gal}_{E / \mathbb{Q}} \\
\pi \neq \pi^{\sigma}}} \sum_{\substack{p \leq x \\
\text { splits } \\
\text { completely }}} \frac{\log ^{2} p}{p} \phi\left(\frac{\log p}{m \log x}\right) \\
& \times \sum_{v \mid p}\left(\sum_{1 \leq j_{1} \leq m} \alpha_{\pi}\left(j_{1}, v\right)\right)\left(\sum_{1 \leq j_{2} \leq m} \bar{\alpha}_{\pi^{\sigma}}\left(j_{2}, v\right)\right)+O(1) .
\end{aligned}
$$

The estimation of (9.4) is the same as in the first case, following (9.2) and (9.3):

$$
=a m^{2}\left(\log ^{2} T\right) \int_{0}^{1 / m} v \phi(v) d v+O(\log T) .
$$


On the other hand, the estimation of (9.5) is done by Lemma 7.4; we get $O(\log T)$. Consequently,

$$
\sum_{p \leq T} \frac{\left|c_{\pi}(p)\right|^{2}}{p} \phi\left(\frac{\log p}{L}\right)=a m^{2}\left(\log ^{2} T\right) \int_{0}^{1 / m} v \phi(v) d v+O(\log T)
$$

and

$$
\begin{aligned}
& \sum_{\gamma_{1}, \cdots, \gamma_{n}} h_{1}\left(\frac{\gamma_{1}}{T}\right) \cdots h_{n}\left(\frac{\gamma_{n}}{T}\right) f\left(\frac{L \gamma_{1}}{2 \pi}, \cdots, \frac{L \gamma_{n}}{2 \pi}\right) \\
= & \frac{\kappa(\mathbf{h})}{2 \pi} T L \ell^{n} \Phi(0, \cdots, 0) \\
& +\frac{\kappa(\mathbf{h})}{2 \pi} T L \sum_{1 \leq r \leq n / 2} \frac{n ! \ell^{n-2 r} a^{r}}{2^{r} r !(n-2 r) !} \int_{\mathbb{R}^{r}}\left|v_{1}\right| \cdots\left|v_{r}\right| \\
& \times \Phi\left(v_{1}, \ldots, v_{r},-v_{1}, \ldots,-v_{r}, 0, \ldots, 0\right) d v_{1} \cdots d v_{r}+O(T) .
\end{aligned}
$$

This proves Theorem 3.1.

Appendix. Proof of Hypothesis $\mathbf{H}$ for $G L(3)$ over $E$. Now we prove Hypothesis $\mathrm{H}$ over $E$ for $m=3$. This was proved in [RudSar] over $\mathbb{Q}$. We follow their approach closely. Since the series on the right side of (5.1) converges absolutely for Re $s>1$, we know that for any $\delta>0$

$$
\sum_{p, k} \sum_{v \mid p} k f_{p}^{2} \frac{\log ^{2} p}{p^{k f_{p}(1+\delta)}}\left|\sum_{j=1}^{3} \alpha_{\pi}^{k}(j, v)\right|^{2}<\infty
$$

Taking $k=1$, we get

$$
\sum_{p} \sum_{v \mid p} \frac{\log ^{2} p}{p^{f_{p}(1+\delta)}}\left|\sum_{j=1}^{3} \alpha_{\pi}(j, v)\right|^{2}<\infty .
$$

On the other hand, as in [RudSar], p.283, we can have $\left|\alpha_{\pi}(2, v)\right|=1$ and $\left|\alpha_{\pi}(1, v)\right|=1 /\left|\alpha_{\pi}(3, v)\right| \geq 1$, because the central character of $\pi$ is assumed to be unitary. Therefore

$$
\begin{aligned}
& \left|\sum_{j=1}^{3} \alpha_{\pi}^{k}(j, v)\right|^{2} \ll \sum_{j=1}^{3}\left|\alpha_{\pi}(j, v)\right|^{2 k} \\
\ll & \sum_{j=1}^{3}\left|\alpha_{\pi}(j, v)\right|^{2 k} \leq\left(\left|\sum_{j=1}^{3} \alpha_{\pi}(j, v)\right|+4\right)^{2 k} \ll 1+\left|\sum_{j=1}^{3} \alpha_{\pi}(j, v)\right|^{2 k} .
\end{aligned}
$$


Consequently

$$
\begin{aligned}
& \sum_{p} \frac{\log ^{2} p}{p^{k f_{p}}} \sum_{v \mid p}\left|\sum_{1 \leq j \leq 3} \alpha_{\pi}^{k}(j, v)\right|^{2} \\
\ll & \sum_{p} \sum_{v \mid p} \frac{\log ^{2} p}{p^{k f_{p}}}+\sum_{p} \sum_{v \mid p} \frac{\log ^{2} p}{p^{k f_{p}}}\left|\sum_{j=1}^{3} \alpha_{\pi}(j, v)\right|^{2 k} .
\end{aligned}
$$

The first sum on the right side of (A.2) is $\leq \ell \sum_{p}\left(\log ^{2} p\right) / p^{k}<\infty$ when $k \geq 2$. Applying the bound in (4.3) to $\left|\sum_{j=1}^{3} \alpha_{\pi}(j, v)\right|^{2 k-2}$, we see that the last sum in (A.2) is

$$
\ll \sum_{p} \sum_{v \mid p} \frac{\log ^{2} p}{p^{f_{p}(1+(k-1) / 5)}}\left|\sum_{j=1}^{3} \alpha_{\pi}(j, v)\right|^{2}<\infty,
$$

by (A.1). Therefore Hypothesis $\mathrm{H}$ is true for $m=3$.

Acknowledgments. The second author wishes to acknowledge support from the Obermann Center for Advanced Studies, the University of Iowa.

\section{REFERENCES}

[ArtClo] J. Arthur and L. Clozel, Simple Algebras, Base Change, and the Advanced Theory of the Trace Formula, Annals of Math. Studies, no. 120, Princeton Univ. Press, Princeton, 1989.

[Bor] A. Borel, Automorphic L-functions, Proc. Symp. Pure Math. 33 (1979), part 2, 27-61.

[GelLapSar] S.S. Gelbart, E.M. Lapid, and P. Sarnak, A new method for lower bounds of L-functions, C.R. Acad. Sci. Paris, Ser. I 339 (2004), 91-94.

[GelShah] S. Gelbart and F. Shahidi, Boundedness of automorphic L-functions in vertical strips, Journal Amer. Math. Soc. 14 (2001), 79-107.

[GodJac] R. Godement and H. Jacquet, Zeta functions of simple algebras, Lecture Notes in Math. 260, Springer-Verlag, Berlin, 1972.

[JacPSShal1] H. Jacquet, I.I. Piatetski-Shapiro, and J. Shalika, Conducteur des réprésentations du groupe linéaire, Math. Ann. 256 (1981), 199-214.

[JacPSShal2] H. Jacquet, I.I. Piatetski-Shapiro, and J. Shalika, Rankin-Selberg convolutions, Amer. J. Math. 105 (1983), 367-464.

[Kim] H. Kim, Functoriality for the exterior square of $G L_{4}$ and the symmetric fourth of $G L_{2}$, J. Amer. Math. Soc., 16 (2003), 139-183.

[KimSar] H. Kim and P. Sarnak, Appendix: Refined estimates towards the Ramanujan and Selberg conjectures, Appendix to [Kim].

[KimShah] H. Kim and F. Shahidi, Cuspidality of symmetric powers with applications, Duke Math. J., 112 (2002), 177-197. 
[Land] E. Landau, Über die Anzahl der Gitterpunkte in gewissen Bereichen, Gött. Nachr. (1915), 209-243.

[Lang] R.P. Langlands, Base Change for $G L_{2}$, Annals of Math. Studies, no. 96, Princeton Univ. Press, Princeton, 1980.

[LiuWangYe] Jianya Liu, Yonghui Wang, and Yangbo Ye, A proof of Selberg's orthogonality for automorphic L-functions, preprint.

[LiuYe1] Jianya Liu and Yangbo Ye, Superposition of zeros of distinct L-functions, Forum Math., 14 (2002), 419-455.

[LiuYe2] Jianya Liu and Yangbo Ye, Weighted Selberg orthogonality and uniqueness of factorization of automorphic L-functions, to appear in Forum Math.

[LiuYe3] Jianya Liu and Yangbo Ye, Selberg's orthogonality conjecture for automorphic L-functions, to appear in Amer. J. Math.

[LuoRudSar] W. Luo, Z. Rudnick, and P. Sarnak, On the generalized Ramanujan conjecture for $G L(n)$, Proc. Symp. Pure Math. 66 (1999), part 2, 301-310.

[MoeWal] C. Moeglin and J.-L. Waldspurger, Le spectre résiduel de $G L(n)$, Ann. Sci. École Norm. Sup. (4) 22 (1989), 605-674.

[Mor1] C.J. Moreno, Explicit formulas in the theory of automorphic forms, Lecture Notes Math. vol. 626, Springer, Berlin, 1977, 73-216.

[Mor2] C.J. Moreno, Analytic proof of the strong multiplicity one theorem, Amer. J. Math., 107 (1985), 163-206.

[Mur1] M. Ram Murty, Selberg conjectures and Artin L-functions, Bull. Amer. Math. Soc. 31 (1994), 1-14.

[Mur2] M. Ram Murty, Selberg's conjectures and Artin L-functions II, Current trends in mathematics and physics, Narosa, New Delhi, 1995, 154-168.

[Rog] J.D. Rogawski, Functoriality and the Artin conjecture, Proc. Symp. Pure Math. 61 (1997), 331-353.

[RudSar] Z. Rudnick and P. Sarnak, Zeros of principal L-functions and random matrix theory, Duke Math. J. 81 (1996), 269-322.

[Sar] P. Sarnak, Non-vanishing of L-functions on $\mathfrak{R}(s)=1$, Shalika's 60th Birthday, preprint, 2003.

[Sel] A. Selberg, Old and new conjectures and results about a class of Dirichlet series, in Collected Papers, vol. 2, Springer, Berlin 1991, 47-65.

[Ser] J.-P. Serre, Letter to J.-M. Deshouillers (1981).

[Shah1] F. Shahidi, On certain L-functions, Amer. J. Math., 103 (1981), 297-355.

[Shah2] F. Shahidi, Fourier transforms of intertwining operators and Plancherel measures for $G L(n)$, Amer. J. Math., 106 (1984), 67-111.

[Shah3] F. Shahidi, Local coefficients as Artin factors for real groups, Duke Math. J., 52 (1985), 973-1007. 
[Shah4] F. Shahidi, A proof of Langlands' conjecture on Plancherel measures; Complementary series for p-adic groups, Ann. Math., 132 (1990), 273-330.

Department of Mathematics, Shandong University, JiNAN 250100, CHINA.

jyliu@sdu.edu.cn

Department of Mathematics, The University of Iowa, IowA City, IowA 52242-1419, USA.

yey@math.uiowa.edu 